\title{
Review
}

\author{
S. S. Mester \\ H. Benaroya \\ Department of Mechanical and \\ Aerospace Engineering \\ Rutgers The State University of \\ New Jersey

\section{Periodic and Near-Periodic Structures}

New Brunswick, NJ

\begin{abstract}
Extensive work has been done on the vibration characteristics of perfectly periodic structures. This article reviews the different methods of analysis from several fields of study, for example solid-state physics and civil, mechanical, and aerospace engineering, used to determine the effects of disorder in one-dimensional (1-D) and 2-D periodic structures. In the work examined, disorder has been found to lead to localization in I-D periodic structures. It is important to understand localization because it causes energy to be concentrated near the disorder and may cause an overestimation of structural damping. The implications of localization for control are also examined. (C) 1995 John Wiley \& Sons, Inc.
\end{abstract}

\section{INTRODUCTION}

The dynamic behavior of structures can be very complex. However, it is important to have a basic understanding of structural behavior to ensure a safe, efficient, and economical design. Aircraft components, turbine blades, satellite antenna dishes, and large space structures are examples of just some of these structures. Typically, their dynamic behavior is found utilizing finite element analysis. For large structures, such as aircraft fuselages and turbine blades, this method is computationally costly. However, because these structures are composed of substructures, which are designed to be identical and uniformly joined to form a repetitive pattern, these structures can be categorized as periodic structures. When the geometric and material periodicities are utilized in the mathematical modeling, the dynamic behavior can be represented as waves propagating through the structure. This allows the application of a well developed body of knowledge about wave propagation. The amount of computation for periodic structures will depend only on the complexity of a substructure, not the number of substructures, thus reducing the computation necessary to analyze the dynamics of a structure.

However, in actual structures, the periodic properties deviate from the ideal. This departure from the ideal has been observed to cause localization. When localization occurs, energy is confined near the disorder and the dynamic behavior of the structure changes. This has several consequences. The localization may be misinterpreted as dissipative damping instead of spatial damping, which may lead to design errors causing unexpected structural failure. Localization can also cause modes to disappear. Therefore, any control scheme such as modal control becomes either ineffective or destabilizing. Indeed, the use and understanding of localization for structural applications may be an efficient means to achieve vibration isolation for a specific section of a

Received July 27, 1993; Accepted June 21, 1994.

Shock and Vibration, Vol. 2, No. 1, pp. 69-95 (1995)

(C) 1995 John Wiley \& Sons, Inc.

CCC 1070-9622/95/020069-27 
structure. Using wave propagation methods, the behavior of disordered structures can be effectively studied.

A broad review of the literature covering many aspects of periodic and near-periodic structures is given. Several approaches are given to analyze the dynamic behavior of periodic and near-periodic structures. Most of the methods use the periodic nature of the structure to reduce the amount of computation required to find the solution. The review points out that structures with slight deviations from a perfectly periodic pattern may behave drastically different than predicted by the periodic methods. Qualitatively, the criteria has been found to be the ratio between the amount of disorder and coupling strength. Although this criteria has been identified, work still needs to be done so that localization can be predicted quantitatively.

\section{VIBRATIONS AND WAVES IN PERIODIC STRUCTURES}

Work from several different fields, such as solidstate physics and civil, mechanical, and aerospace engineering, related to the dynamics of periodic and nonperiodic structures is reviewed. The methods used to study solid-state crystal lattices are applied to analyze the dynamics of periodic and near-periodic structures. The review first looks at vibrations and wave propagation in periodic structures and then examines near-periodic structures. It ends with a look at the control aspects of these structures.

\section{Relation Between Vibrations and Waves}

The two different ways of representing structural motion, vibrations and waves, can be derived from each other (Feather, 1961). A vibrating string is used to illustrate the equivalence. Given the equation for the transverse displacement of a vibrating string

$$
y=A \sin \frac{\pi x}{l} \cos \frac{\pi}{l} \sqrt{\frac{T}{m}} t,
$$

the procedure of resolution of vectors is used to derive the equations of wave motion. Equation (1) is resolved into two components of amplitude $A / 2$ each. The components lead and lag the original motion of Eq. (1) by the same angle, $\Delta$. The form of the wave solution is assumed to be

$$
\begin{aligned}
y= & y_{1}+y_{2} \\
= & \frac{A}{2} \cos \left(\frac{\pi}{l} \sqrt{\frac{T}{m}} t+\Delta\right) \\
& +\frac{A}{2} \cos \left(\frac{\pi}{l} \sqrt{\frac{T}{m}} t-\Delta\right) .
\end{aligned}
$$

Applying the parallelogram law,

$$
\begin{aligned}
y^{2} & =\left|y_{1}\right|^{2}+\left|y_{2}\right|^{2}-2\left|y_{1}\right|\left|y_{2}\right| \cos (\pi-2 \Delta) \\
& =2\left(\frac{A}{2}\right)^{2}+2\left(\frac{A}{2}\right)^{2} \cos 2 \Delta,
\end{aligned}
$$

where $y=A \sin (\pi x / l)$. Thus,

$$
A^{2} \sin ^{2} \frac{\pi x}{l}=A^{2} \cos ^{2} \Delta,
$$

so,

$$
\Delta=\frac{\pi x}{l}-\frac{\pi}{2},
$$

because cosine leads sine by an angle of $\pi / 2$. This results in

$$
\begin{aligned}
& y_{1}=\frac{A}{2} \sin \frac{\pi}{l}\left(\frac{T}{m} t+x\right) \\
& y_{2}=-\frac{A}{2} \sin \frac{\pi}{l}\left(\frac{T}{m} t-x\right) .
\end{aligned}
$$

This represents two different simultaneous weave motions that sum to an equivalent of the vibrating motion.

\section{One-Dimensional (1-D) Perfect Structures}

A periodic structure is characterized by identical substructures that are joined to each other in a consistent manner to form the overall structure. There are many methods of analysis that can be utilized to find the dynamic response of these structures, such as finite element methods (FEMs), transfer matrix analysis, wave propagation analysis, and continuum modeling. The computational effort required for the analysis of the dynamic behavior of such structures can be greatly reduced by taking advantage of the periodicity of the structure.

The FEM can be used either by itself or in combination with another method. In classical 
vibration analysis, the modes are computed for the entire structure directly. However, for large structures many elements are needed for accuracy, increasing the computation cost. Thus, the size of the structure and/or accuracy of the analysis is limited by the amount of computational power available. When the periodicity of a structure is utilized, a repeated cell may be modeled and analyzed by the FEM to obtain its properties that are then used by a periodic method to find the behavior of the overall structure. Thus, unlike the direct method, only one substructure needs to be analyzed regardless of the overall size of the structure. Denke, Eide, and Pickard (1975) use the FEM to determine the mechanical impedance matrix of a substructure,

$$
A=K-\omega^{2} M+j \omega C .
$$

From the equation of motion,

$$
A X=F_{\mathrm{ext}},
$$

the transfer matrix is found as follows. First the substructure's interior degrees of freedom are eliminated by replacing them with equivalent loads and an equivalent impedance matrix at the substructure's boundaries. Partitioning the resulting equation of motion as follows

$$
\left[\begin{array}{cc}
A_{\mathrm{B}_{\mathrm{H}}} & A_{\mathrm{B}_{\mathrm{Ir}}} \\
A_{\mathrm{B}_{\mathrm{rl}}} & A_{\mathrm{B}_{\mathrm{rr}}}
\end{array}\right]\left\{\begin{array}{l}
X_{\mathrm{B}_{1}} \\
X_{\mathrm{B}_{\mathrm{r}}}
\end{array}\right\}=\left\{\begin{array}{l}
F_{\mathrm{ext}_{\mathrm{B}_{1}}} \\
F_{\text {ext }_{\mathrm{B}_{\mathrm{r}}}}
\end{array},\right.
$$

where the subscript, B, indicates that the values are for the substructure's boundaries and the subscripts, 1 and $r$ indicate the left or right side of the substructure, respectively (see Fig. 1). It turns out that the transfer matrix is

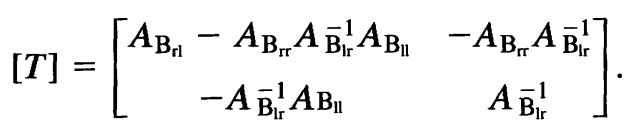

Bardell and Mead (1989a,b) used a special FEM, known as the hierarchical FEM, to find the stiffness and mass matrices of a flat rectangular panel and a cylindrically curved rectangular panel. In this case, the substructure is a super element in the hierarchical FEM.

Several articles have been written that examine the application of the transfer matrix method to the problem of dynamic analysis of periodic structures. The implementation of the method can involve some numerical difficulties in com-

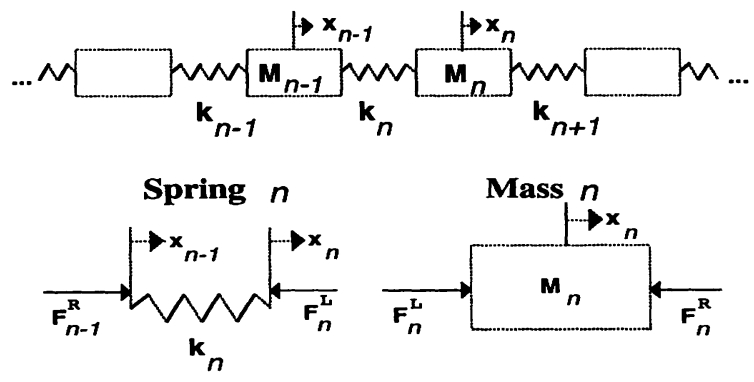

Figure 1 A linear chain oscillator used to develop the transfer matrix method.

puting the chain product of the transfer matrix. Lin and McDaniel (1969) point out that the multiplication of many matrices can lead to significant roundoff errors. Instead of directly multiplying the matrices, the Cayley-Hamilton theorem is used. In general, the transfer matrix of a system with a $k$-dimensional state vector is $k \times k$. Other properties of the transfer matrix is that it also is a transfer matrix in the other direction. The existence of an inverse also ensures that the transfer matrix is nonsingular. It is found that

$$
|T|=\left|T^{-1}\right|=1,
$$

which implies that the characteristic equation of the transfer matrix and its inverse are the same; they have the same set of eigenvalues. Because for every eigenvalue, $\lambda_{i}$, of $[T]$ there is a corresponding eigenvalue, $\bar{\lambda}_{i}=1 / \lambda_{i}$ of $[T]^{-1}$, it can be concluded that the set of eigenvalues of the transfer matrix is pairwise reciprocal. Lin and McDaniel (1969) used a periodically supported Bernoulli-Euler beam to illustrate this method. The state vector of the beam is four-dimensional (4-D) so the transfer matrix is $4 \times 4$. However, they mention that an extensive catalog of basic transfer matrices has been compiled by Pestel and Leckie (1963), which can be used in the implementation of this method to other structures. It was noted that the transfer matrix method is limited to 1-D structures and some two-dimensional (2-D) structures in which the spatial variable can be separated.

Meirovitch and Engels (1977) use the $Z$-transform to find the solution of a transfer matrix problem. A structure with a 2-D state vector, displacement, and force, is assumed to illustrate the method. Arbitrary external forces applied at the boundaries between the elements can be handled by adding an external force vector, $f_{i}^{*}$, such that 


$$
x_{i+1}^{L}=A_{i} x_{i}^{L}+f_{i}^{*} .
$$

The $Z$-transform is defined as

$$
x(z)=Z\left[x_{i}\right]=\sum_{i=0}^{\infty} x_{i} z^{-1}
$$

Taking the $Z$-transform of Eq. (12) and rearranging, results in

$$
x(z)=Z[z I-A]^{-1} x_{0}+[z I-A]^{-1} f^{*}(z)
$$

The response, $x_{i}$, can be found by taking the inverse $Z$-transform and rearranging as such,

$$
x_{i}=\phi_{i} x_{0}+\sum_{k=0}^{i-1} \phi_{i-k-1} f_{k}^{*}
$$

where $\phi_{i}$ is the "fundamental matrix" of the system. It is shown that $\phi_{i}=A^{i}$. Computing $\phi_{i}$ from this method encounters the same numerical difficulties noted by Lin and McDaniel (1969). In the $Z$-transform method, the roundoff error is overcome by using the Leverrier algorithm to solve the following inverse $Z$-transform relation

$$
Z^{-1}\left\{z[z I-A]^{-1}\right\}=\phi_{i}
$$

After some manipulation, this yields

$$
\phi_{i}=Z^{-1}\left[\sum_{j=1}^{2 n} \frac{z}{z-z_{j}} X_{j}\right]
$$

where $z_{j}$ are the eigenvalues of matrix $[A]$ and

$$
\begin{aligned}
X_{j} & =\lim _{z \rightarrow z_{j}}\left[\left(z-z_{j}\right) X(z)\right] \\
& =\frac{\sum_{l=0}^{2 n-1} z_{j}^{2 n-l-1} H_{l}}{\sum_{l=0}^{2 n-1}(2 n-l) \theta_{l} z_{j}^{2 n-l-1}},
\end{aligned}
$$

in which $H_{l}$ and $\theta_{l}$ are determined by the Leverrier algorithm. This results in a response of

$$
x_{i}=\sum_{j=1}^{2 n} X_{j}\left(z_{j}^{i} x_{0}+\sum_{k=0}^{i-1} z_{j}^{i-k-1} f_{k}^{*}\right)
$$

Note that for a semiinfinite structure $i \rightarrow \infty$, so roots, $z_{j}$, with a magnitude greater than 1 cause Eq. (19) to diverge unless they are discarded. This requires that only half of the eigenvalues need to be found. For finite structures, the fact that the eigenvalues occur in reciprocal pairs allows the Leverrier algorithm to reduce the order of the characteristic equation by a factor of two. In addition, this method does not require the eigenvectors of matrix $A$, only the eigenvalues, thus simplifying the computation and increasing the accuracy of the solution. From the analysis of an illustrative example, it is shown that a finite structure with heavy damping can be treated as semiinfinite because the response is virtually unaffected by the motion at the other end. The finite structure in the example is composed of 20 substructures and the response was computed for two boundary conditions: fixed-free and freefree. Engels (1980) addresses the divergence of Eq. (19) due to eigenvalues with magnitudes greater than one. The method of approach is the same as in Meirovitch and Engels (1977). However, using the spectral form of matrix $A$,

$$
A=\sum_{j=1}^{2 n} X_{j} z_{j}
$$

the response of a semiinfinite structure with a load at the left end is found to be

$$
x_{i}=\left\{\begin{array}{l}
u_{i} \\
p_{i}
\end{array}\right\}=\left\{\begin{array}{l}
\left(\sum_{j=1}^{n} X_{11 j} z_{j}^{i}\right)\left(\sum_{j=1}^{n} X_{21 j}\right)^{-1} f_{0} \\
\left(\sum_{j=1}^{n} X_{21 j} z_{j}^{i}\right)\left(\sum_{j=1}^{n} X_{21 j}\right)^{-1} f_{0}
\end{array}\right\} .
$$

The response of an infinite structure due to an interior load is

$$
u_{i}=\sum_{j=1}^{n} X_{12 j}^{T} z_{j}^{l-1} f_{l-1}^{* \prime}, \quad p_{i}=-\sum_{j=1}^{n} X_{11 j}^{T} z_{j}^{l-1} f_{l-1}^{* \prime} .
$$

For multiple loads the response is obtained by superposing the appropriate combination of the two solutions above. The solutions are found in terms of the known boundary conditions.

Engels and Meirovitch (1978a) formulated a method that computes the natural frequencies of a structure in a systematic way. It can also handle arbitrary excitations. The approach is a combination of the $Z$-transform method explained in the two previous articles and Holzer's method. It is shown that

$$
x_{i}=A^{i} x_{0}=\phi_{i} X_{0} .
$$

The above equation can be partitioned as 


$$
x_{N}=\left[\begin{array}{ll}
\phi_{N_{11}} & \phi_{N_{12}} \\
\phi_{N_{21}} & \phi_{N_{22}}
\end{array}\right] x_{0} .
$$

This is rewritten as

$$
\begin{aligned}
& u_{N}=\phi_{N_{11}} u_{0}+\Phi_{N_{12}} p_{0}, \\
& p_{N}=\phi_{N_{21}} u_{0}+\phi_{N_{22}} p_{0} .
\end{aligned}
$$

Depending on whether the structure has freefixed, fixed-free, free-free, or fixed-fixed end conditions, the following yields the natural frequencies of the system:

$$
\operatorname{det} \phi_{N_{r s}}=0 \text {. }
$$

The roots of the characteristic determinant are found by Muller's method (1956). The method is iterative and requires only one evaluation of the characteristic determinant per iteration. However, the derivatives of the characteristic determinant are not needed. Once a natural frequency is found it is divided out of the characteristic determinant and the next frequency is found. Then from the following relation

$$
x_{i, k}=\phi_{i}\left(\lambda_{k}\right) x_{0, k}, \quad i=1,2, \ldots, N,
$$

the $k$ th mode is fully given. Note that the internal forces $p_{i, k}$ are also obtained. With the natural frequencies and mode shapes found, the procedure of modal analysis can compute the response of the structure due to arbitrary loads. This method was originally developed for undamped structures, however it should be possible to apply this method to damped structures with some adjustments to the method.

Engels and Meirovitch (1978b) have modeled continuous systems as periodic structures. This builds on the $Z$-transform method. However, as the substructure becomes smaller the influence of the mass matrix vanishes when using the $Z$ transform as derived previously. Thus the algorithm has been reworked with the assumption of substructures with small lengths that approach zero. The method obtains the response of damped and undamped systems subject to harmonically distributed loads. This covers the current work done on the dynamic analysis of periodic structures using transfer matrices based on the Holzer method.

Extensive work has been done on the wave receptance theory. The contributions of Mead and others are briefly reviewed. Mead (1973) has studied energy flow in 1-D multicoupled structures using the receptance method. A multicoupled system is characterized by identical bays that are joined by more than one coordinate or degree of freedom. Letting $R$ denote the $R$ th free wave, the energy of the wave is expressed as follows

$$
E=\frac{1}{2} \operatorname{Re}\left[i \omega\left[F_{\mathrm{l}}^{*}\right]\left[\alpha_{\mathrm{ll}}-e^{u_{R}}\left(\beta_{\mathrm{sy}}+\beta_{\mathrm{ss}}\right)\right]\left\{F_{\mathrm{l} R}\right\}\right],
$$

where $\left[F_{I R}^{*}\right.$ is the transposed conjugate of $\left\{F_{I R}\right\}$ and

$$
\begin{aligned}
& \beta_{\mathrm{sy}}=\frac{1}{2}\left(\alpha_{\mathrm{lr}}+\alpha_{\mathrm{rl}}\right) \\
& \beta_{\mathrm{ss}}=\frac{1}{2}\left(\alpha_{\mathrm{lr}}-\alpha_{\mathrm{r} l}\right) .
\end{aligned}
$$

This gives the time-averaged energy crossing the junction between two elements. Energy is only propagated when $\mu_{R}$ is imaginary.

For imaginary propagation constants, a close approximation of the possible frequencies is obtainable by an approach that uses Rayleigh's principle. The approximate frequencies are given by

$$
\omega_{R j}^{2}=\frac{\left[q_{R_{j}}^{* T}[K]\left\{q_{R_{j}}\right\}\right.}{\left[q_{R_{j}}^{* T}[M]\left\{q_{R_{j}}\right\}\right.} .
$$

Note that this method requires guessed values for $\left\{q_{R_{j}}\right\}$, but the error of $\omega_{R_{j}}^{2}$ is only second order. It can be shown that this method has stationary properties, which allows for the exact frequencies to be found after some rearrangement and differentiation.

Mead (1975a) goes on to investigate the reflections of waves impinging on the ends of finite monocoupled structures. First, the concept of a characteristic wave receptance,

$$
\alpha_{\omega}=q_{1} / F_{1}=\alpha_{11}-e^{\mu} \alpha_{1 \mathrm{lr}},
$$

is introduced. This is the ratio of the complex displacement at a coupling point in the infinite periodic structure to the complex (harmonic) force at that point when a single characteristic free wave travels through the system. Then for a finite structure with $N$ bays the end receptances are 


$$
\begin{aligned}
\alpha_{A A} & =\alpha_{\mathrm{ll}}-\alpha_{\mathrm{lr}} \frac{\sinh (N-1) \mu}{\sinh N \mu} \\
\alpha_{B A} & =\alpha_{\mathrm{lr}} \frac{\sinh \mu}{\sinh N \mu} .
\end{aligned}
$$

The natural frequencies of a system with both ends free are found by letting $\alpha_{A A} \rightarrow \infty$ and with both ends fixed the natural frequencies are found by letting $\alpha_{A A}=0$. The above work is extended by Mead (1975a,b) to multicoupled structures with $n$ coupling points between substructures. The method is the same as in the preceding reference, with the exception of the reflection of a characteristic wave at a boundary. For one incident wave there can be $n$ reflected waves. However, in most structures the nonpropagating waves vanish before they reach the other end, so computing the natural modes and frequencies from the remaining waves is greatly simplified. Mead (1986) investigated a new approach to analyzing infinite uniform 1-D structures supported periodically. In this approach, the supports are considered to be a "phased array" of forces and moments that can be analyzed using point direct receptances of an unconstrained structure. Based on the support conditions, the propagation constant can be found using equations relating displacements to forces, rotations to forces, displacements to moments, and rotations to moments. The total response of the structure to a convected pressure field is the sum of the response of the unconstrained structure and the phased array response, which is imposed by the loading and the constraints. Mead and Yaman (1990) use the method of phased arrays to examine finite uniform Euler-Bernoulli beams. The method uses the receptance function of the infinite structure. Then the free waves are found using the boundary conditions at the ends of the structure and at the supports.

In a continuum approach, the structure is modeled as an equivalent substitute continuum. Then using the properties of the equivalent continuum, the motion of the structure is predicted. Noor, Anderson, and Greene (1978) developed a method for developing continuum models for repetitive beam- and plate-like lattices with arbitrary configurations subjected to static, thermal, and dynamic loadings. The continuum models of the repeating elements are characterized by their thermoelastic strain and kinetic energies that allow the equations of motion and the constitutive relations to be derived. This is accomplished by expanding the nodal displacements of a typical element in a Taylor series and then equating the potential and kinetic energies of the lattice to the potential and kinetic energies of the continuum model. Using the assumption that the strain at the element boundaries must be the same for both elements, the dimensionality of the problem is reduced. Then the continuum model properties are derived so that the potential and kinetic energies of the lattice and the continuum model are the same. Once the properties are found, the continuum model's behavior can be analyzed and should directly correspond to the lattice's behavior. This method is especially useful for lattice structures with a large number of panels where either a small number of finite elements are used for their analysis or analytic solutions are obtained. The method has been extended to generate the analytic expressions for the substitute continuum properties of a large repetitive lattice structure. Application to beam-like and doublelayered plate-like lattices are illustrated by Noor and Mikulas (1988).

\section{2-D Perfect Structures}

Plane wave motion can be analyzed for 2-D structures that have periodicity in two orthogonal directions, although not necessarily with the same period. The propagation constants in the $x$ and $y$ direction of a plane wave inclined at angle $\theta$ to the $x$-axis of a plane periodic structure oriented parallel to the $x-y$ plane are

$$
\mu_{x}=k l_{x} \cos \theta, \text { and } \mu_{y}=k l_{y} \sin \theta,
$$

where $l_{x}$ and $l_{y}$ are the dimensions of the 2-D substructure and $k$ is the propagation constant in the direction of the wave. The Rayleigh quotient method can be used to determine the frequencies for a given propagation constant, in a similar way as for the 1-D case. The Rayleigh quotient method should be applicable to the three-dimensional (3-D) case based on its physical interpretation (Mead, 1973).

Mead, Zhu, and Bardell (1988) looked at a flat plate with an orthogonal array of uniform beams as reinforcement. Plane wave motion was considered with different propagation constants in the orthogonal directions. Hierarchical finite elements were used to set up the equations of motion for the substructure, which are solved as an eigenvalue problem. The structure has passbands and stop bands. 
Bardell and Mead (1989a,b) studied a cylindrical superstructure consisting of cylindrically curved rectangular panels with orthogonal stiffeners. The propagation of plane waves were analyzed by examining the different propagation constants in the axial and circumferential directions. The propagation constants were found by solving the governing equations for free vibration as an eigenvalue matrix problem. The more realistic case of a point source is more complicated to analyze and so far has not been attempted. This would produce elliptical wave fronts.

Anderson (1981) has estimated the buckling load of a lattice with repetitive geometry. Applications examined were an isogrid cylinder, a three element truss column, and a polygonal ring. The analysis of these 3-D structures was approached by assembling the substructure from a network of substructural members, such as rods, cables, and rings.

As an extension of this work, Anderson investigated (1982) the vibration of prestressed shelland beam-like periodic lattice structures. Each member of the structure was represented by an exact dynamic stiffness matrix that was then used to solve an eigenvalue problem of the same order as the number of degrees of freedom for a node. The analysis was developed for a cylindrical beam truss, beginning with the relation of forces and displacements of node 0 to its neighboring nodes

$$
\begin{aligned}
F_{0}=R U_{0}+S U_{j}, & \quad(j \text { represents neighboring node } j)
\end{aligned}
$$

where

$$
\begin{aligned}
F_{i}^{T} & =\left[P_{1}, P_{2}, P_{3}, M_{1}, M_{2}, M_{3}\right]_{i} \\
U_{i}^{T} & =\left[u_{1}, u_{2}, \psi_{1}, \psi_{2}, \psi_{3}\right]_{i} .
\end{aligned}
$$

The force and momentum along each local coordinate is $P$ and $M$, respectively. The matrices, $R$ and $S$, are $6 \times 6$ matrices that are modified finiteelement stiffness matrices such that the exact behavior of all vibration modes of the element are represented without requiring intervening nodes. Then using finite-element transformation matrices to write the equilibrium equations at a node in terms of the displacement of a typical node and the displacements of adjacent nodes, the equation is written as

$$
\sum_{j}\left(G_{j} U_{0}+H_{j} U_{j}\right)=\omega^{2} U_{0},
$$

where $G_{j}$ and $H_{j}$ are the transformation of $R$ and $S$ from local to global coordinates. The mass matrix $J$ includes only the concentrated masses at the nodes. Because the structure is periodic,

$$
U=U_{0} e^{i \pi x / \lambda} e^{i n \phi}
$$

satisfies the equilibrium equations above. The axial coordinate is represented by $x$ and $\phi$ is the circumferential coordinate. The mode shape has $n$ circumferential waves and an axial half-wavelength $\lambda$. Using Eq. (38) to eliminate the displacements at the adjacent nodes in the equilibrium equation results in

$$
\left(K-\omega^{2} J\right) U_{0}=0,
$$

where $K$ is the assembled global stiffness matrix as implemented by Anderson (1981). The desired eigenvalues are obtained by a Gaussian elimination method (Wittrick and Williams, 1971). The implementation of the theory developed by Anderson is complicated due to the difficulties in dealing with boundary conditions because his method does not make any provisions for different boundary conditions.

Nagem and Williams (1989) used transfer matrices and joint coupling matrices to compute the natural frequencies of a lattice structure. The matrices can also be used to analyze wave propagation with some further work. The method is accurate and more efficient than FEM for situations that require many high frequency modes.

\section{Problems with Circular Geometries}

There are many different geometric configurations of periodic structures that have been examined. The three primary ones are beam, circular assemblies, and lattices (trusses). Mead and Bardell $(1986,1987)$ examined a cylindrical shell with discrete axial stiffeners and a cylindrical shell with periodic circumferential stiffeners. These were treated as 1-D periodic structures. The propagation constants were found by an exact technique that finds the displacements in closed form. Stop and passbands were found. In a damped structure, the attenuation constants are all increased; however, some are more so than others. The pattern of the variation of the propagation constants is greatly changed. Narrow propagation zones can disappear due to moderate damping. The damping is hysteretic with the value of $\eta=0.1$ where $\eta$ is the structural 
loss factor. The effects of the various stiffeners are that as the spacing between stiffeners is decreased, the propagation zones are shifted to higher frequency bands and the attenuation constants become smaller. There are four pairs of propagation constants for each structure: that is, eight possible traveling waves. The motion parallel to the stiffeners is restricted to a sinusoidal variation while orthogonal to the stiffeners it can be arbitrary.

Bardell and Mead (1989a,b) extended their study to cylindrical shells with orthogonal stiffeners. These structures are 2-D and the method of analysis is discussed above. Also investigated were thin cylinders with stringer stiffeners axially and frame stiffened circumferentially using the same method. Their experiments agree with the theory.

\section{Biperiodic Structures}

McDaniel and Carroll (1982) examined space structures that are biperiodic. Biperiodic structures have two types of substructural assemblies that alternate throughout the structure. Biperiodic beam analysis has been done for two support cases: knife edge supports and elastic biperiodic supports. The approach is similar to that of Ghatak and Kothari (1972). The method is based on the use of a chain of oscillators, in which the masses alternate between $m_{1}$ and $m_{2}$. The transfer matrix method is used to relate the slopes and moments between substructures of a beam. Different end conditions are simply postmultiplied in matrix form. Some 2-D problems can be solved as above if separation of variables can be applied to the system. The results yield frequency propagation bands in which the natural frequencies are usually found. The frequency bands of periodic structures are divided into two bands for biperiodic structures.

In application, perfectly periodic structures do not exist due to manufacturing and construction tolerances. Therefore, small random imperfections need to be considered. This leads to the study of near-periodic structures that has followed a number of approaches: the receptance method on systems with one disorder, perturbation approaches, and stochastic methods (See Mester, 1992). It has been found that imperfections in the periodicity of the structure can lead to a phenomena called localization. The following gives an overview of this work.

\section{WAVE PROPAGATION IN NEAR-PERIODIC STRUCTURES}

Near-periodic structures are "periodic" structures that contain either one element that is not identical to the others, known as a single disorder, or many elements that deviate from an average repeated element by a small amount, known simply as disordered. Mead and Bansal (1978) developed a general theory for monocoupled periodic systems with a single disorder. Wave receptance methods were used. In a perfect system the forced waves would propagate without obstruction, but a disorder also causes characteristic free waves to be generated from the disorder. The response is the sum of both. The ratios of the forces at the ends of the disordered substructure are

$\frac{F_{\mathrm{rA}}}{F_{\mathrm{i} A}}=\frac{\left(\alpha_{\mathrm{w}+}-\alpha_{\mathrm{AA}}\right)\left(\alpha_{\mathrm{w}+}+\alpha_{\mathrm{BB}}\right)+\beta_{\mathrm{AB}} \beta_{\mathrm{BA}}}{\left(\alpha_{\mathrm{w}+}+\alpha_{\mathrm{AA}}\right)\left(\alpha_{\mathrm{w}+}+\alpha_{\mathrm{BB}}\right)-\beta_{\mathrm{AB}} \beta_{\mathrm{BA}}}$

$\frac{F_{\mathrm{tA}}}{F_{\mathrm{i} A}}=\frac{\left(2 \alpha_{\mathrm{w}+} \beta_{\mathrm{BA}}\right)}{\left(\alpha_{\mathrm{w}+}+\alpha_{\mathrm{AA}}\right)\left(\alpha_{\mathrm{w}+}+\alpha_{\mathrm{BB}}\right)-\beta_{\mathrm{AB}} \beta_{\mathrm{BA}}}$,

where the subscripts A and B represent the left and right side of the disordered element. The disturbance is taken to impinge upon the left side of the nonperiodic substructure. The subscripts $i, r$, and $t$ indicate incident, reflected, and transmitted. The subscript $w+$ implies that the receptance function is for positive going waves. These expressions are in terms of the end receptances of the disorder, $\beta_{m n}$, and the characteristic wave receptance of the periodic system, $\alpha_{m n}$. This method has been extended by Mead and Lee (1984) to analyze 1-D systems with a disorder that is a cluster of elements which is periodic, but is not the same periodicity as the rest of the system.

Ziegler (1977) uses Floquet waves to examine the behavior of periodic and disordered composite materials. The method is based on a transfer matrix. The state vector consists of the displacements of the left and right going waves. The analysis is continued by examining the complex state ratio, which is the ratio of a left going wave to the right going wave, through conformal mapping.

Disorder in a system, such as the spinal column, where geometry as well as mass and stiffness change, can also be viewed as a perfectly periodic system that has been perturbed. Meirovitch and Engels (1978) derived an efficient perturbation technique to get the response of a 
disordered "periodic structure." The $Z$-transform method as explained above is extended by a perturbation approach to obtain the response of almost periodic structures. Here, the transfer matrix $A$ is no longer constant, but can be assumed to be

$$
A_{i}=A_{i}^{(0)}+\varepsilon A_{i}^{(1)}+\varepsilon^{2} A_{i}^{(2)}+\cdots,
$$

where $A_{i}^{(0)}$ is a constant matrix and $\varepsilon$ is a small parameter. Then

$$
\vec{x}_{i}=\vec{x}_{i}^{(0)}+\varepsilon \vec{x}_{i}^{(1)}+\varepsilon^{2} \vec{x}_{i}^{(2)}+\cdots \cdot
$$

Inserting Eqs. (42) and (43) into Eq. (12) and equating like powers of $\varepsilon$, results in

$$
\vec{x}_{i+1}^{(l)}=A^{(0)} \vec{x}_{i}^{(l)}+\vec{f}_{i}^{(l)}, \quad l=0,1,2, \ldots,
$$

where

$$
\vec{f}_{i}^{(0)}=\vec{f}_{i}^{*}, \quad \vec{f}_{i}^{(l)}=\sum_{k=1}^{l} A_{i}^{(k)} \vec{x}_{i}^{(l-k)}, \quad l=1,2, \ldots
$$

Proceeding as with the periodic solution, the response of the system can be found. The first order approximation is usually sufficient for most problems. A 20 substructure axial single degree of freedom system was worked. The results for both perturbed and unperturbed were found, and both have similar responses except that the unperturbed system consistently has a slightly smaller displacement. This method can accommodate variations in all system parameters as long as the variations remain the same.

Huang (1982) examined disordered structures by modeling them with periodic random parameters. In this approach, the systems parameters such as mass, $m_{x}(\theta)$, and local frequencies, $q^{2}(\theta)$ and $p^{2}(\theta)$, are expressed as random variables, each with a mean value and a variance of unity. This analysis is developed using an undamped set of blades on a disk. The equation of motion is

$$
\begin{aligned}
-q_{0}^{2}\left[(1+\eta Q) X^{\prime}\right]^{\prime}+ & {\left[p_{0}^{2}(1+\xi P)\right.} \\
& \left.-\omega^{2}(1+\zeta M)\right] X=0 .
\end{aligned}
$$

The functions $M, P$, and $Q$ are random functions corresponding to $m_{x}, p$, and $q$. The subscript 0 denotes the mean value of the parameter. The Greek letters represent small parameters equal to the standard deviations of the corresponding random variables. The natural frequencies and mode shape functions are found through a perturbation method using only the first-order terms

$$
\begin{aligned}
\omega^{2} & =\lambda^{2}+\xi \mu^{2}+\eta \nu^{2}+\zeta \rho^{2}+\cdots \\
X(\theta) & =y(\theta)+\xi u(\theta)+\eta v(\theta)+\zeta w(\theta)+\cdots .
\end{aligned}
$$

Substituting Eq. (47) into Eq. (46), then collecting like orders of $\xi, \eta$, and $\zeta$, the natural frequencies and mode shapes are found by the use of Fourier series expansion to be

$$
\begin{aligned}
\omega_{m}^{2}= & \left(p_{0}^{2}+m^{2} q_{0}^{2}\right)+\xi p_{0}^{2} \int_{-\pi}^{\pi} P y_{m}^{2} d \theta \\
& +\eta q_{0}^{2} \int_{-\pi}^{\pi} Q y_{m}^{\prime 2} d \theta-\zeta \lambda_{m}^{2} \int_{-\pi}^{\pi} M y_{m}^{2} d \theta .
\end{aligned}
$$

The mean value of $\omega_{m}^{2}$ is

$$
E\left[\omega_{m}^{2}\right]=p_{0}^{2}+m^{2} q_{0}^{2},
$$

and the standard deviation is a small quantity, on the same order as $\xi, \eta$, and $\zeta$. The normal modes are shown to be orthogonal. Two results are found that differ from deterministic solutions. The first result is that the phase angles of the normal modes are no longer arbitrary, but they are independent of initial conditions. The other result is that normally resonance only occurs when $k \Omega=\omega_{m}$ and $k=m$, where $\Omega$ is the exciting frequency and $k$ is the number of the harmonic of $\Omega$. With the random parameter modeling, the above still holds; however, weak resonance occurs when $k \Omega=\omega_{m}$ and $k \neq m$.

Soong and Bogdanoff (1963) looked at the effects of disorder on the natural frequencies of a linear chain. The analysis was conducted using both a deterministic and probabilistic approach. In the deterministic approach, an additional term was added to represent a deterministic change in a parameter. It was concluded that unless the inertial and elastic parameters are exactly known, the highest few natural frequencies may deviate considerably from the predicted values. The practical significance of this conclusion is that little confidence can be placed in the calculated values of the top few natural frequencies in view of the difficulty in estimating accurately the elastic constants in most engineering systems. On the other hand, the demonstrated stability of 
the lowest few natural frequencies with respect to parameter variation increases our confidence in our ability to estimate these frequencies with considerable accuracy. For the probabilistic approach, the parameters are represented as a mean value plus a random variable with zero mean and a small variance with respect to unity. The arrived-at conclusions are the same as for the deterministic case. The probability distribution for the higher natural frequencies are spread over a relatively broad range of frequencies, such that neighboring natural frequencies distributions overlap. Thus, little confidence can be put into the prediction of the higher natural frequencies.

Soong and Bogdanoff (1964) extended the above analysis to examine the impulsive admittance and the frequency response of the disordered chain. The impulsive admittance function was expanded as a series of statistically independent random variables. It was shown that the mean of the disordered admittance function is the same as the deterministic admittance function. However, the standard deviation of the admittance function increases as the damping constant decreases. Thus, an accurate prediction for the behavior of lightly damped structures is not possible if the parameter values are not exact. The mean of the frequency response deviates increasingly from the deterministic frequency response as the input frequency increases, but the standard deviation of the random model decreases as input frequency increases.

\section{Localization}

In disordered structures, a phenomenon called localization may occur in which energy is confined near the disorder and the dynamic behavior of the structure changes. It is important to examine this effect for several reasons. This energy confinement can be mistaken for structural damping during physical testing. The results of the testing may be used to determine material properties for analysis and design. leading to erroneous designs. The confinement can possibly be used in a creative way to isolate critical areas of the structure from disturbances.

Hodges (1982) used two examples, coupled pendula and a vibrating string, to study the effects of localization in disordered systems. In the system of coupled pendula, the disorder arises from the length of the pendula deviating from a uniform length. In the vibrating string, disorder is induced by constraining the string at irregular in- tervals. Localization does not allow waves to propagate far from the source through energy confinement, not dissipation. It was shown that pendula of the same natural frequency have normal modes of behavior even as the coupling strength decreases. But as the pendula become more and more disordered, the coupling must become stronger and stronger to maintain the normal modes. If the coupling strength is weak, then the pendula begin acting independently, barely affecting their neighbor. A string with masses and springs attached at irregular intervals was also examined. The wavelength was assumed to be much smaller than the fluctuation in interval spacing. The equations derived were for one degree of freedom. The analysis was done in terms of wave propagation and reflection from structural irregularities.

Bendiksen (1987) conducted a theoretical and numerical investigation into the possible localization or confinement of vibratory modes in large space structures due to structural imperfections. Localization causes a global modal amplitude to become confined to a local region in the structure. Localization has serious effects on the control of structures. The study shows that mode localization is most likely to occur in structures with many weakly coupled substructures. Space structures with high model densities are in this category; thus it is important to include the effect of structural imperfections and disorder in designing their control systems. Examples of large space structures are solar energy collectors, solar sails, large astronomical telescopes, communication antennas, and space station structures. The approach uses ideas and results from different fields such as solid-state physics, solid mechanics, and dynamics. In dynamic stability and control, it was shown that disorder can actually be stabilizing. However, a serious consequence of localization is that it destroys the regular features of the modes, like regular spacing of the nodal points and lines, and the sinusoidal amplitude modulation. The amplitude and phase relationships of sensor signals would become radically different from their expected values. Thus a control system tuned to the ideal system would attempt to control an essentially unknown system. Preliminary results also indicate that the identification problem may also be a major challenge. This makes it difficult to apply adaptive control. The example used to illustrate the approach was a circular periodic monocoupled 1-D structure like a bladed rotor. It has circular peri- 
odicity and global periodicity due to the fact that the first and last substructures are adjacent. It is noted that structural coupling extended beyond adjacent substructures. The solution for a perfectly ordered dish was obtained.

Imperfections were then introduced into the example of the dish. The stiffness matrix $K$ was changed by an amount $\Delta K$ where $\Delta K / K$ is a random variable with a uniform probability function on an interval [0, 0.05]. For $K_{c} / K=1$ and $K_{h} / K=10$, strong localization occurred. The location of the peaks could not be anticipated by a cursory inspection of the distribution of the imperfections, and different modes localized at different substructures. The recognizable features of the perfect modes, like nodal lines, were obliterated.

For multi degree of freedom systems, perturbation methods were used. The perturbation equations are

$$
\begin{aligned}
{[\tilde{M}] } & =[M]+\varepsilon[m] \\
{[\tilde{K}] } & =[K]+\varepsilon[k],
\end{aligned}
$$

where $\varepsilon \ll 1$ and $[m]$ and $[k]$ are the same order as the unperturbed matrices. The perturbation method gives the insight that imperfections cause modes that were originally orthogonal to become coupled. Small changes cause the system to behave differently, thus the perturbation method is not valid because it violates the assumption that the change is small. The ratio $\varepsilon / K_{c}$ is used to circumvent the violation of the assumption. Localization increases as the ratio of the disorder strength, $\varepsilon$, to interstructure coupling strength, $K_{c}$, increases. The ratio, $\varepsilon / K_{c}$, is more important than just the degree of disorder, $\varepsilon$, itself. Bendiksen (1987) suggested that significant localization results from structural irregularities within normal manufacturing tolerances.

Pierre, Tang, and Dowell (1987) used a perturbation method to study localization that is found in disordered multispan beams. The method found strongly localized modes without a global eigenvalue analysis of the entire system. Criteria that predicts the occurrence of strong localization was found. For the first group of modes, strong localization occurs if the relative passband width of the tuned beam is of the order of, or smaller, than the relative spread in the frequencies of the individual spans. It was suspected that if localization does not occur in the first two modes, then it will not occur in the higher modes; but if it occurs in these modes then it will occur in the higher modes as well, but the severity will not increase. The accuracy of the method was experimentally verified. It was discovered that small disorders in weakly coupled structures will cause localization.

In structures with close eigenvalues, Pierre (1988) has shown that small structural irregularities result in both strong localization of mode shapes and abrupt veering away of the loci of the eigenvalues. Perturbation methods for the eigenvalue problem were applied to predict the occurrence of strong localization and eigenvalue loci veering, which turned out to be two effects of the same phenomenon.

$$
\begin{aligned}
\lambda_{i} & =\lambda_{0 i}+\delta \lambda_{i}+\delta^{2} \lambda_{i} \\
w_{i} & =w_{0 i}+\delta w_{i}+\delta^{2} w_{i} \\
\delta \lambda_{i} & =\left\langle w_{0 i}, l w_{0 i}\right\rangle \varepsilon \\
\delta w_{i} & =\left\{\sum_{j \neq i} \frac{\left\langle w_{0 j}, l w_{0 i}\right\rangle}{\lambda_{0 i}-\lambda_{0 j}} w_{0 i}\right\} \varepsilon .
\end{aligned}
$$

A criterion for localization is found to be

$$
\left|\lambda_{0 r}-\lambda_{0 s}\right| \leq O\left(\left|\left\langle w_{0 s}, l w_{0 r}\right\rangle \varepsilon\right|\right),
$$

where $\left\langle w_{0 i}, l w_{0 i}\right\rangle$ is an inner product, $\varepsilon$ is a small perturbation, and $l$ is an operator that represents the equation of motion. Equation (52) indicates a violation of the perturbation assumptions of small variations about $w_{0 i}$, because $\delta w_{i}$ has an order of magnitude of one or greater. Thus for closely spaced eigenvalues strong localizations most likely occur. Perturbations are taken to the second order, because the first-order method is not sensitive enough to small disorder $(\varepsilon \leq 10 \%)$.

The transmission of steady-state harmonic vibration from a local source of excitation in a nearly periodic assembly of monocoupled, multicomponent mode substructures was examined by Cha and Pierre (1991) using probabilistic methods. A transfer matrix formulation was used to derive analytical expressions for the localization factor in the limiting cases of strong and weak modal coupling. The localization factor increases rapidly with each successive passband. Typically the transition from weak to strong localization occurs from one passband to the next and severe vibration confinement is unavoidable at high frequencies, even for a small disorder.

Cai and Lin (1990) developed a new perturbation scheme using probability theory to calculate 
the average attenuation rate with respect to the distance of the transmission, called the localization factor. Structural damping was also considered. The method is general and permits successive improvements in accuracy. This was achieved by taking the reflections from nearby disordered cells successively and substituting the ensemble average for the sequential average of certain statistical properties of the cells on the basis of spatial ergodicity. They compare well with Monte Carlo simulations.

A statistical investigation of the effects of disorder on the dynamics of 1-D nearly periodic structures was presented by Pierre (1990). The problem of vibration propagation was examined when disorder was introduced. Analytical expressions for the localization factors (the exponential decay constants) were obtained in the two limiting cases of weak and strong internal coupling ratio and the excitation frequency. Both modal and wave propagation descriptions were used. The perturbation results were verified by Monte Carlo simulations. The phenomena of weak and strong localization was evidenced. Although the former has little effect on the dynamics of most structures, the latter was shown to be of significant importance in structural dynamics. Weak internal coupling and small disorder has drastic effects on dynamic properties due to strong localization.

Pierre (1990) studied the localization phenomenon for a generic model of 1-D nominally periodic system. An in depth examination of the frequency dependence of localization effects in the weak and strong regimes was given. The distinction between weak and strong localization effects was classified. The localization factors for limited cases were evaluated by stochastic perturbation methods. For strong coupling and large disorder, localization is insignificant for engineering structures because of the effects of damping and boundary conditions. The dependence of the localization factor upon frequency, coupling, and disorder was analyzed. The validity of the analytical results were verified by Monte Carlo simulations. Weak localization occurs with strong (finite) internal coupling and weak disorder in which amplitude decay per site is small. Weak localization is of little concern because the decay is small, boundary conditions can effect the small localization factors significantly and obscure any notable disorder effects, and light damping is at least on the same order making weak localization of little interest, although in structures that con- tain hundreds of substructures, weak localization can become significant. Strong localization happens in weakly coupled, weakly disordered systems. For strong localized vibrations only a few substructures participate and are more significant in engineering structures.

Experiments have been conducted that confirm the existence of the localization phenomenon in actual structures. The localization of the response of a 12 rib satellite antenna structure was predicted fairly well by Levine-West and Salama (1992) using the MSC/NASTRAN model that used the measured structural dimensions from off the actual experimental structure. Bouzit and Pierre (1993) conducted a physical investigation of a simply supported beam, which gave good agreement with theoretical analysis. These results indicated that the importance of considering localization is not just academic.

Another way of looking at the dynamic behavior of a structure is power flow or power propagation analysis, which is closely related to wave propagation analysis. Instead of examining the wave motion at the different frequencies, the energy flow is considered.

Keane and Price (1989) examine monocoupled and undamped structures via several different methods to obtain a better insight of the dynamic behavior of plated structures with welded stiffeners. All the methods, wave, modal, receptance, and finite element, were found able to give similar results. This highlights the importance of correctly modeling the subject of analysis as compared to the method of analysis. Localization due to a single disorder is explained as a result of a shift in the passbands of a disordered element. The shifted passband admits natural frequencies that are now in the stop band of the rest of the elements. Therefore, the modes of these frequencies become localized as they are attenuated traveling through the stop bands of the periodic elements. They suggested that Anderson localization for most engineering structures has little effect and for those that are effected, such as highly random or weakly coupled structures, should not be analyzed using periodic or nearperiodic methods.

Zhong and Williams (1991) investigated localization for high frequency modes of disordered structures using an analogy between computational structural mechanics and optimal control. The simplectic matrix approach was used to obtain the eigenvalues of the structure as well as the limits of the pass bands. 


\section{POWER PROPAGATION IN NETWORKS}

The following studies developed methods to analyze vibrational energy propagation in structural networks. The methods are generalized to be applicable to any arbitrary structural network. However, as in other analysis methods, periodic structures simplify the analysis.

Mead (1985) investigated the power flow in Timoshenko beams and examined the power transmission and reflection at constraints. Von Flotow (1986a) combined several mathematical methods to form a systematic approach in analyzing the disturbance propagation in a structural network. The concepts used were traveling wave modes and scattering matrices from microwave circuit analysis. Continuum models of periodic members were used. Members were described in the frequency domain by the propagation coefficients of their intrinsic wave modes, while junctions were represented by frequency dependent wave-mode reflection and transmission coefficients grouped in a junction scattering matrix. The component impulses were calculated by a combination of analysis and the fast Fourier transform algorithm. The convolution of the impulse responses gave the network response. Parallel processing will be needed in order to get real time solutions.

Miller and von Flotow (1989) looked at structural networks as the assembly of slender 1-D members. Each member was defined by two junctions. Junctions may be terminations or the interconnections of many members. Elastic disturbance propagation and power flow in networks were considered. Spatially local models were assembled into a global frequency domain description. The global response description was then examined to find the local and global power flow. The method can be used to determine the disturbance transmission paths. The method also helps the designer choose and evaluate control procedures for the structure.

Structural component models of wave propagation were assembled into a global model and power flow at all levels was investigated. Component net power flow provided local information about the dissipation and energy transmission of members and junctions. The combinations of wave modes present in a member or junction was determined by the global network behavior. Total network power flow showed which components have the most vibrational energy. The power relations showed the physical paths that dominate the extraction of energy. The global modeling method led to detailed response predictions. The method is limited to networks of 1-D members but could be extended to higher dimensions.

Cai and Lin (1991) examined power flow in structures. The members were treated as a multichannel waveguide, and the structure was considered as a network. Three analytical tools were used: individual waveguides, junctions where the waveguides meet, and boundaries where either displacements or forces are zero or a mixture. All three may be represented by a wave scattering matrix that relates incoming waves to outgoing waves in a structural element.

Zhang and Zhang (1991) investigated ways of reducing the vibrational energy flow in a periodically supported beam. The relation between the location of the excitation and the transmitted energy flow was examined. The location where the excitation would minimize the transmitted energy can be found for different propagation bands. The power is split in half, regardless of the excitation location, and each half flows in an opposite direction from the excitation. The effect of damping is that power is reduced near the bounding frequencies of the odd propagation zones.

\section{STRUCTURAL CONTROL WITHOUT IMPERFECTION}

Once the dynamics of these periodic structures are understood, the next step is to apply this understanding to control the behavior of the structures to meet the requirements of the task they were designed to accomplish. This can be done either actively or passively. Active control requires sensors, actuators, and controllers. Passive control is designed into the system so that the desired behavior is inherent. It appears that some form of active control will be required for imperfectly periodic structures. Here, some representative work on the control of waves in structures is summarized.

Von Flotow and Schafer (1986) approached the vibrational control of a hanging beam by two methods that turned out to be very similar. They are velocity (direct) feedback and wave absorbing controllers. The wave absorbing controllers were designed to minimize the reflection of energy. The experimental results indicated that 
more work needs to be done in order to realize these theoretical wave-absorbing controllers.

Von Flotow (1986b) investigated active control of propagating disturbances by modifying the natural disturbance propagation path. The disturbance energy was shunted into unimportant areas of the structure or absorbed by "wave absorbers." This method though is not practical for low frequency or large amplitude disturbances.

Pines and von Flotow (1988) conducted an analytical and experimental investigation to determine if the propagation of bending waves along a uniform beam can actively be blocked. Strain gauge sensors drove piezoceramic actuators as a dynamic compensator, designed in the frequency domain, but independent of the boundary conditions. The experimental results were promising although not completely successful.

Bennighof and Meirovitch (1989) compared two methods of actively suppressing traveling waves in structures. When higher modes were damped out passively, independent modal-space control could be concentrated on the lower modes. But when the modal frequencies were closely spaced, direct feed back control was better because of the large number of modes that needed to have energy dissipated. The model method is a global method, while the direct feedback method is local. However, both methods tend to concentrate the actuator forces near the localized disturbance. It should be noted that the modal method can only control as many modes as there are actuators.

A frequency dependent cost function including both power flow and control effort was minimized by Miller, Hall, and von Flotow (1989) to find the optimal feedback compensation for structural waveguides at junctions. Wave models try to control all frequencies that propagate through a structure; modal models single out the frequencies that correspond to modes. Wave models cannot show global resonant behavior, but they show that critical locations can be isolated. Also weave modeling requires fewer degrees of controller freedom to implement control. Wave modeling can be used to create control schemes to locally suppress vibration and create dynamic isolation.

Hagwood, Chung, and von Flotow (1990) modeled the effects of dynamic coupling between a structure and an electrical network through the piezolectric effect. This laid the groundwork for optimizing the use of piezolectric materials as sensors and actuators in active structural con- trol. The mechanical and electrical coordinates were obtained by Hamilton's principle using a Rayleigh-Ritz formulation.

These articles indicate several control approaches. Although the approaches are general, their application to periodic structures should capitalize upon the unique features of these structures such as reduced computation and passive filtering. Additionally, it is expected that the effects of imperfections, that is, localization, can have a serious impact on the propagation of energy through a structure. Thus, any effective control strategy must account for localization effects.

This work was prepared in partial fulfillment of the requirements for the degree of Doctor of Philosophy of the first author. The work was performed under the support of the Federal Aviation Administration Technical Center.

\section{APPENDIX: SELECTED ABSTRACTS ON PERIODIC AND NEAR PERIODIC STRUCTURES}

Anderson, M. S., 1981, "Buckling of Periodic Lattice Structures," AIAA Journal, Vol. 19, pp. 782-788.

Equations are developed for the buckling of a general lattice structure that has repetitive geometry. Equilibrium at a typical node is expressed using finite-element techniques, and the only assumption is that the response is periodic. The stiffness matrix is based on the exact solution of the beam column equation; thus, accurate results are obtained for complex buckling behavior that would require a very large system of equations using conventional techniques. The present method requires the eigenvalues of only a $6 \times 6$ determinant. The results are used to study the buckling of isogrid cylinders, three-element truss columns, and polygonal rings. Details of the analysis including expressions for all terms in the governing stability determinant are given.

Anderson, M. S., 1982, "Vibration of Prestressed Periodic Lattice Structures," AIAA Journal, Vol. 20, pp. 551-555.

Equations are developed for vibration of general lattice structures that have repetitive geometry. The method of solution is an extension of a previous article for buckling of similar structures. The theory is based on representing each member of the structure with the exact dynamic stiffness matrix and tak- 
ing advantage of the repetitive geometry to obtain an eigenvalue problem involving the degrees of freedom at a single node in the lattice. Results are given for shell-like and beam-like lattice structures and for rings stiffened with tension cables and a central mast. The variation of frequency with external loading and the effect of local member vibration on overall modes is shown.

Bardell, N. S., and Mead, D. J., 1989, "Free Vibration of an Orthogonally Stiffened Cylindrical Shell I: Discrete Line Simple Supports," Journal of Sound and Vibration, Vol. 134, pp. 29-54.

The hierarchical finite element method is used to establish the stiffness and mass matrices of a cylindrically curved rectangular panel. Some natural frequencies and modes of two such panels, each with different boundary conditions, are then determined. Excellent agreement is found between this work and that of other investigators. These stiffness and mass matrices are then combined with the periodic structure theory to analyze an orthogonally stiffened cylindrical shell. This analysis is formulated for a "plane wave" type of motion characterized by different propagation constants in the axial and circumferential directions. The governing equations of free vibrations are then solved as a matrix eigenvalue problem for the frequencies at which particular waves will propagate. Results are presented in the form of phase-constant surface stacks, and clearly show the qualitative effects of varying the major shell parameters $b / a, R / a$, and $R / h$.

Bardell, N. S., and Mead, D. J., 1989, "Free Vibration of an Orthogonally Stiffened Cylindrical Shell II: Discrete Line Simple Supports," Journal of Sound and Vibration, Vol. 134, pp. 55-72.

A thin, cylindrical shell is considered, stiffened axially by equipitched, identical stringers and stiffened circumferentially by equipitched, identical frames. Generality of stringer and frame section is allowed. The structure is analyzed as a two-dimensional periodic structure by using wave propagation techniques in conjunction with the hierarchical finite element method. Results are presented in the form of phase-constant surfaces plotted against frequency. It is shown that free wave motion can propagate in the infinite structure from zero frequency. A small frequency band has been identified in which predominantly flexural waves cannot propagate. Experiments performed on a one-quarter scale fuselage model confirm the main findings of the theoretical analysis.

Bendiksen, O. O., 1987, "Mode Localization Phenomena in Large Space Structures," AIAA Journal, Vol. 25, pp. 1241-1248.
An analytical and numerical study is presented that investigates the possibility of localization or confinement of vibratory modes in large space structures. These structures belong to a class of periodic structures that have recently been shown to be sensitive to periodicity-breaking disorder or imperfections. When localization occurs, the modal amplitude of a global mode becomes confined to a local region of the structure, with serious implications for the control problem. The results of this study show that mode localization is most likely to occur in structures consisting of a large number of weakly coupled substructures. Certain large space structures with high modal densities fall in this category, and it is therefore important to include the effect of structural imperfections and disorders when designing control systems for shape or directional control of such structures.

Bennighof, J. K., and Meirovitch, L., 1989, "Active Suppression of Traveling Waves in Structures," Journal of Guidance, Control, and Dynamics, Vol. 12, pp. 555-567.

The article is concerned with the control of traveling waves in structures. Two approaches are used: independent modal-space control (IMSC) and direct feed back control. Direct feed back control is shown to be more suitable for problems in which a large number of higher modes require control. IMSC is shown to be more suitable when the number of modes in need of control is not large or when inherent damping provides passive control, suppressing the higher modes. One fact worthy of notice is that although IMSC represents a global approach compared to direct feed back control, the actuator forces still tend to concentrate near localized disturbances such as traveling waves.

Bouzit, D., and Pierre, C., 1993, “Experimental Investigation of Vibration Localization in Disordered Multispan Beams,' Collection of Technical PapersAIAA/ASME Structures, Structural Dynamics and Materials conference, Vol. 3, pp. 1565-1577.

The results of an experimental investigation of the effects of span length disorder on the dynamics of a monocoupled, multispan beam are reported. Two experimental specimens are considered: a nominally periodic 12-span beam with equal spacing between simple supports, and the corresponding disordered beam that features slightly randomly spaced supports. Experimental results demonstrate that the transmission of vibration that takes place within the frequency passbands of the periodic beam is greatly hindered when span length randomness is introduced. The spatial localization of both the mode shapes and the steady-state harmonic response to an end excitation is observed in the disordered 12-span 
beam, especially for frequencies that lie within the second passband. Good quantitative agreement is obtained between experimental results and theoretical findings for both ordered and disordered configurations. Furthermore, an experimental estimation of the localization factor yields satisfactory agreement with the theoretical result for an infinite multispan beam. This work reports one of the first systematic experiments carried out to demonstrate the occurrence of vibration localization in nearly periodic structures.

Cai, G. Q., and Lin, Y. K., 1991, "Localization of Wave Propagation in Disordered Periodic Structures," AIAA Journal, Vol. 29, pp. 450-456.

A structure designed to be spatially periodic in its configuration cannot be exactly periodic due to material, geometric, and manufacturing variabilities. Such variabilities are random, and their presence (often referred to as disorder) can reduce the ability of the structure to transmit waves from one location to another. The phenomenon is known as vibration confinement or localization. A new perturbation scheme is developed on the basis of probability theory to calculate the average exponential decay rate of wave transmission with respect to the distance of transmission, called the localization factor. Account is also taken of structural damping. The new scheme permits successive improvement of accuracy, making it applicable to either weak, strong, or moderate localization. Moreover, the analysis is based on a generic periodic structure; thus, it is not restricted to a specific set of governing equations. These are achieved by taking into account reflections from nearby disordered cell units successively and substituting the ensemble average for the sequential average of certain statistical properties of the cell units on the basis of spatial ergodicity. Application of the method is illustrated by an example, and the results are compared with Monte Carlo simulations.

Cai, G. Q., and Lin, Y. K., 1991, "Wave Propagation and Scattering in Structural Networks," Journal of Engineering Mechanics, Vol. 117, pp. 1555-1574.

A theoretical procedure is described for the dynamic response analysis of engineering structures composed of interconnected slender members. Each structural member is treated as a multichannel waveguide, and the entire structure is treated as a network of such waveguides. Three types of analytical building blocks are then discussed: an individual waveguide, a junction where several waveguides intersect, and a boundary where either zero displacements or zero forces or their combination must be imposed. It is shown that the properties of each type of building block may be characterized by a wavescattering matrix that relates the incoming waves to the structural element in question and the outgoing waves from the structural element. The use of wavescattering matrices guarantees that numerical computation is always stable, because the computation follows the direction of wave propagation, and the wave amplitude can only decrease with the propagation distance. The proposed analytical procedure is efficient in computation and it yields accurate results, especially if motions at specific locations on a structure are required.

Cha, P. D., and Pierre, C., 1991, "Vibration Localization by Disorder in Assemblies of Monocoupled, Multimode Component Systems," Journal of Applied Mechanics, Vol. 58, pp. 1072-1081.

Disorder in nominally periodic engineering structures results in the localization of the mode shapes to small geometric regions and in the attenuation of waves, even in the passbands of the corresponding perfectly periodic system. This article investigates, via probabilistic methods, the transmission of steady-state harmonic vibration from a local source of excitation in nearly periodic assemblies of monocoupled, multicomponent mode substructures. A transfer matrix formulation is used to derive analytical expressions for the localization factor (the rate of exponential decay of the vibration amplitude) in the limiting cases of strong and weak model coupling. The degree of localization is shown to increase with the ratio of disorder strength to modal coupling. The increase is nearly parabolic for small values of this ratio, and logarithmic for large values. Furthermore, the localization factor increases rapidly with the passband number. Typically, the transition from weak to strong localization occurs from one pass band to the next, and severe vibration confinement is unavoidable at high frequencies, even for small disorders.

Denke, P. H., Eide, G. R., and Pickard, J., 1975, "Matrix Difference Equation Analysis of Vibrating Periodic Structures," AIAA Journal, Vol. 13, pp. 160166.

The matrix difference equation (MDE) method for sound transmission and forced vibration analysis of damped periodic structures is presented. A periodic structure is defined as a string of identical substructures, such as a segment of aircraft fuselage that has identical bays between circumferential frames. The finite element method is applied to the substructure to provide a mechanical impedance matrix. A matrix difference equation is derived from the impedance matrix, based upon conditions of equilibrium and compatibility at substructure boundaries. The difference equation is reduced in order by eliminating force variables and introducing substructure displacement modes. A solution is found by calculating 
eigenvalues and eigenvectors of a related characteristic equation. The result is a closed form expression in the longitudinal coordinate. The method is general and applicable to complex structures, because of the finite element basis. Results of an application to aircraft engine duct vibration are included.

Engels, R. C., 1980, "Response of Infinite Periodic Structures,' Journal of Sound and Vibration, Vol. 69, pp. 181-197.

The response of infinite and semiinfinite periodic structures to harmonic loads is investigated. The method developed requires the eigenvalues of the transfer matrix of a typical substructure. Consequently, the algorithm is capable of analyzing an infinite periodic structure with the same computational effort necessary to analyze a single substructure. Furthermore, the solution is given in terms of known boundary conditions and no eigenvectors of the transfer matrix are required. Several examples are included. Additional simplifications can be obtained when the substructure is symmetric.

Engels, R. C., and Meirovitch, L., 1978, "Response of Periodic Structures by Modal Analysis," Journal of Sound and Vibration, Vol. 56, pp. 481-493.

A periodic structure is a structure consisting of identical substructures, coupled together in identical ways to form the complete system. The undamped response of such a system is derived by using a modal analysis technique. The procedure allows for arbitrary loads and takes full advantage of the periodic properties of the structure. The algorithm is based on a technique previously developed by the authors.

Engels, R. C., and Meirovitch, L., 1978, "Simulation of Continuous Systems by Periodic Structures,' Journal of Applied Mechanics, Vol. 45, pp. 385-392.

Many continuous systems can be approximated by periodic structures (structures consisting of identical substructures, connected to each other in identical manner). An efficient algorithm developed by these authors for the response of periodic structures is adapted to the treatment of continuous systems. The method is capable of deriving the response of damped or undamped systems subject to harmonic distributed loads. The length of the substructure can be made arbitrarily small without increasing the computational effort. Furthermore, the number of degrees of freedom of the substructure can be reasonably large.

Ghatak, A. K., and Kothari, L. S., 1972, An Introduction to Lattice Dynamics, Addison-Wesley Publishing Company, Boston.
This monograph discusses topics in lattice dynamics and its study by neutron scattering experiments. The topics covered include wave propagation in a one-dimensional lattice, quantization of lattice vibrations in one-dimension, elastic constants and waves, the dispersion relation and quantization of lattice vibrations in three-dimensions, and the theory of specific heats of solids. The phenomenological theory of lattice dynamics is also developed and the Debye approximation is justified. Neutron scattering experiments are described to explain how they are used to determine the frequency distribution function and dispersion relation for lattice modes.

Hagood, N. W., Chung W. H., and von Flotow, A., 1990, "Modeling of Piezoelectric Actuator Dynamics for Active Structural Control," Journal of Intelligent Material Systems and Structures, Vol. 1, pp. 327-354.

The article models the effects of dynamic coupling between a structure and an electrical network through piezoelectric effect. The coupled equations of motion of an arbitrary elastic structure with peizoelectric elements and passive electronics are derived. State-space models are developed for the three important cases: direct voltage driven electrodes, direct charge driven electrodes, and an indirect drive case where the piezoelectric electrodes are connected to an arbitrary electrical circuit with embedded voltage and current sources. The equations are applied to the case of a cantilevered beam with surface mounted piezoceramics and indirect voltage and current drive. The theoretical derivations are validated experimentally on an actively controlled cantilevered beam test article with indirect voltage drive.

Hodges, C., 1982, "Confinement of Vibration by Structural Irregularity," Journal of Sound and Vibration, Vol. 82, pp. 411-424.

The propagation of vibrations in structures with some degree of extended disorder (i.e., departure from regularity or strict periodicity extended throughout the structure) is discussed. An account is given of the phenomenon of normal mode localization, caused under certain circumstances by the disorder. This phenomenon means that vibrational energy injected into the structure by an external source cannot propagate to arbitrarily large distances, but is instead substantially confined to a region close to the source. Specifically, it is shown that the steady response of the structure decays exponentially away from the source. This phenomenon, predicted by Anderson in 1958 with applications in solid-state physics in mind, has been of great importance in understanding electrical conduction processes in disordered solids and can be 
very important in acoustical problems as well. In particular, it means that the literature on periodic structures can be misleading in a qualitative, and not just a quantitative, manner. The phenomenon is illustrated in terms of two simple mechanical models, a system of coupled pendula and a vibrating string or bending beam with mass and spring constraints.

Huang, W. hu, 1982, "Vibration of Some Structures with Periodic Random Parameters," AIAA Journal, Vol. 20, pp. 1001-1008.

In a periodic structural system such as blades in a closed packet of turbomachinery, the natural frequencies of the individual blade can be randomly different from one another. This article describes a solution approach for such a periodic structure in which the distributions of blade frequencies are random processes with small standard deviations. A spectral method is suggested to solve for differential equations with random coefficients. The expressions for vibration modes are given, the standard deviations of natural frequencies are estimated, and the results of forced vibration are presented. Some special features of vibration characteristics of this system are shown as well.

Keane, A. J., and Price, W. G., 1989, "On the Vibrations of Mono-Coupled Periodic and Near-Periodic Structures," Journal of Sound and Vibration, Vol. 128, pp. 423-450.

The fundamental equations governing the linear, oscillatory dynamics of structures consisting of repeated similar substructures are examined. The systems considered are monocoupled and undamped, and they are examined by using wave, modal, receptance, and finite element (FE) analyses. These analyses are aimed at providing an improved insight into the vibratory behavior of engineering structures that exhibit periodicity. In particular they are directed toward the plated structures with evenly spaced welded stiffeners that are commonly employed in modern engineering designs (e.g., ships, aircraft, etc.). Results originally derived from studies of the microscopic behavior of the constituent atoms of crystalline solids are used in this work, and some of the differences of emphasis highlighted. The effects of deviations from perfect periodicity are considered, particularly with reference to the dramatic changes in mode shapes that sometimes occur. Nonetheless, it is demonstrated that in the present context, all the methods of analysis used are capable of giving similar results (within anticipated accuracies), indicating the importance of correctly modeling a structure, as compared with the exact details of how the model is then analyzed.
Levine-West, M. B., and Salama, M. A., 1992, "Model Localization Experiments on a Ribbed Antenna," AIAA/ASME/ASCE/AHS/ASC Structures, Structural Dynamics and Materials Conference Technical Papers, Vol. 4, pp. 2038-2047.

The model localization (ML) phenomenon is investigated experimentally and analytically to determine the influence of its parameters. For this purpose, a full-scale 12-rib loosely coupled antenna test bed with small imperfections is dynamically tested for various levels of interrib coupling stiffness and excitation force. The experimental results are described herein. Using a simplified numerical model of the structure, a sensitivity analysis of the modal behavior is also performed. The numerical and experimental results are shown to agree remarkably well, thereby providing conclusive validation of the ML phenomenon on a testbed having the dynamic characteristics of space structures.

Lin, Y. K., and McDaniel, T. J., 1969, "Dynamics of Beam-Type Periodic Structures," ASME-Paper 69Vibr-17.

Analytical technique is developed for determination of frequency response matrix of one-dimensional periodic structure; detailed analysis is carried out for periodic Bernoulli-Euler beams on many elastic supports, a simplified version of the skin-stringer panel system commonly used in flight vehicle designs. It is shown that considerable simplification can be achieved by introducing one of several possible interior singular conditions. Numerical examples are given for an undamped system and for the same system with damper units attached at periodic locations.

McDaniel, T. J., and Carroll, M. J., 1982, "Dynamics of Bi-Periodic Structures," Journal of Sound and Vibration, Vol. 81, pp. 311-335.

In order to gain insight into the dynamics of biperiodic aerospace structures, a variety of one- and two-dimensional biperiodic structures are considered. It is shown that bands in which natural frequencies lie for periodic structures are further subdivided as a consequence of the biperiodicity. Analytical solutions for the modes and frequencies of finite-length, one-dimensional, biperiodic structures are obtained for general boundary conditions. A transmission method is developed to simplify the application of boundary conditions. Some modes are found to occur at frequencies outside the frequency bands predicted for biperiodic structures. Two-dimensional biperiodic crossed beam grillage and truss structures are considered in this study. 
Mead, D. J., 1973, “A General Theory of Harmonic Wave Propagation in Linear Periodic Systems with Multiple Coupling," Journal of Sound and Vibration, Vol. 27, pp. 235-260.

A general theory is presented of harmonic wave propagation in one-dimensional periodic systems with multiple coupling between adjacent periodic elements. The motion of each element is expressed in terms of a finite number of displacement coordinates. The nature and number of different wave propagation constants at any frequency are discussed, and the energy flow associated with waves having real, complex, or imaginary propagation constants is investigated. Kinetic and potential energy functions are derived for the propagating waves and a generalized Rayleigh's quotient and Rayleigh's principle for the complex wave motion have been found.

Mead, D. J., 1975, “Wave Propagation and Natural Modes in Periodic Systems: I-Mono-Coupled Systems," Journal of Sound and Vibration, Vol. 40, pp. 1-18.

Two topics are treated: the relationship between the bounding frequencies of the propagation zones of monocoupled systems and the natural frequencies of the individual elements making up such systems is studied. Properties of free harmonic wave propagation in undamped multicoupled systems are identified for predicting resonant frequencies and response levels in these systems. The concept of characteristic receptance of a free wave in the periodic system is developed in the treatment of the first topic. Two examples of real aerospace structures provide a frame of reference for the discussion of the second topic: a periodic skin-stringer structure and a periodic rib-skin structure.

Mead, D. J., 1975, “Wave Propagation and Natural Modes in Periodic Systems: II-Multi-Coupled Systems, with and without Damping," Journal of Sound and Vibration, Vol. 40, pp. 19-39.

Free waves can propagate through periodic systems only in particular frequency zones. Equations for the bounding frequencies of these zones are obtained in terms of the receptance matrices of the elements of multicoupled systems. The relationship between these frequencies and the natural frequencies of a single element of the system is considered, particular attention being given to elements that are symmetrical. The nature of the characteristic wave motions is studied, and a characteristic receptance matrix for a characteristic wave is defined. This is used to introduce the study of reflection of a characteristic wave from a boundary. The equations governing the reflection process are set up, and used to formulate the equations for the natural frequencies and modes of a finite periodic system with arbitrary boundaries. The modes are represented by superpositions of opposite-going pairs of characteristic waves, in terms of which a simple physical description of the natural wave motion is presented.

Mead, D. J., 1985, "Wave Propagation in Timoshenko Beams," Stronjnicky Casopis, Vol. 36, pp. 556-585.

The work presented in this article is relevant to the problem of high-frequency, short wavelength vibration transmission in beams. If the wavelength is much less than the length of the beam, certain vibration problems can be solved assuming that the beam is of infinite length. The frequency-averaged response to a point harmonic force (or moment), averaged over several resonating modes, is equal to the response of the infinite beam to a point harmonic load (or moment). It is therefore of value to study these latter problems in detail. It is also helpful to understand the nature and mechanism of wave energy transmission in beams. This, in turn, can be used to analyze and understand the reflection and transmission process when an incident wave in a beam impinges on a discontinuity or on a constraint. From this, one can deduce how to prevent wave energy in one region of a beam from being transmitted to more remote regions. All of this has been well established for Euler-Bernoulli beams. It now remains to use the Timoshenko beam theory to study these phenomena over a wider frequency range than was formally valid. These problems will be considered in this article.

Mead, D. J., 1986, “A New Method of Analyzing Wave Propagating in Periodic Structures-Applications to Periodic Timoshenko Beams and Stiffened Plates," Journal of Sound and Vibration, Vol. 104, pp. 9-27.

A response function is found for an infinite, uniform, one-dimensional structure that is subjected to an array of harmonic forces or moments, spaced equidistantly, and that have a constant phase or ratio between any adjacent pair. Receptance functions are derived for these "phased arrays." They are used to set up a general determinantal equation for the propagation constants of the infinite structure when it is made periodic by the addition of an infinite set of regular constraints. They are also used to set up equations for the response of the structure to a convected harmonic pressure field. The method enables the equations for the propagating constants and for the response to convected loading to be set up with much greater facility than by earlier methods. It only requires a knowledge of the response function of the infinite uninterrupted structure under a single-point harmonic force or moment. The gen- 
eral equation for the propagation constants is used to study a simple supported periodic Timoshenko beam, and a parallel plate with periodic beam-type stiffeners. Some calculated propagation constants are presented and discussed. The periodic plate results are relevant to integrally stiffened skins of the type used in airplanes.

Mead, D. J., and Bansal, A. S., 1978, "Mono-Coupled Periodic Systems with a Single Disorder-Free Wave Propagation," Journal of Sound and Vibration, Vol. 61, pp. 481-496.

A general method has been presented for analyzing free harmonic wave propagation through a monocoupled periodic system with a single disorder. Expressions have been derived for the magnitudes of the waves transmitted and reflected by the disorder. These general expressions have then been used to study flexural wave motion through a periodic beam system into which three different types of disorders have been introduced: a beam element of nonperiodic length; a rotary mass at a support; a rotary spring at a support. The disorder always results in reduced transmission of the flexural wave when the frequency is in a frequency propagation zone of the periodic system but the first two disorders may lead to increased transmission in a frequency attenuation zone. Conditions have been identified under which the combined disorder plus periodic system can behave like a resonating spring-mass system, or as a spring-mass damper system. The adverse effects of resonating disorders are pointed out. Qualitative and quantitative analysis based upon computer studies have indicated how disorders can be used most effectively for vibration isolation in existing periodic systems or when designing new systems.

Mead, D. J., and Bardell, N. S., 1986, "Free Vibration of a Thin Cylindrical Shell with Discrete Axial Stiffeners," Journal of Sound and Vibration, Vol. 111. pp. 229-250.

Wave propagation around a cylindrical shell that is reinforced at regular intervals by flexible stiffeners parallel to the shell generator is considered. The shell itself is restricted to a section between two circumferential frames onto which the shell is simply supported. The structure effectively constitutes a one-dimensional periodic system and is analyzed as such. Four degrees of freedom are allowed between each periodic element and equations are set up for the four pairs of propagation constants that characterize the possible wave motions. Symmetric or asymmetric stiffener sections may be accommodated in the analysis together with structural damping. Computed propagation constants are presented for two different stiffener cross-sections, each pitched at two different intervals around the shell. Natural frequencies are calculated for one of these.

Mean, D. J., and Bardell, N. S., 1987, “Free Vibration of a Thin Cylindrical Shell with Periodic Circumferential Stiffeners," Journal of Sound and Vibration, Vol. 115, pp. 499-520.

The theory is developed for obtaining the propagation constants of a thin uniform cylindrical shell, periodically stiffened by uniform circular frames of general cross-section. The free wave motion is analyzed and the stop and passbands of free wave motion in the structure are located. Hysteretic damping is included. The natural frequencies of two stiffened finite cylindrical shells are deduced. The relative effects of the frame cross-section and pitch on the free vibration characteristics of the whole structure are discussed.

Mead, D. J., and Lee, S. M., 1984, "Receptance Methods and the Dynamics of Disordered One-Dimensional Lattices," Journal of Sound and Vibration, Vol. 92, pp. 427-445.

The method of receptance analysis is used to set up a frequency equation for the free vibration modes of a one-dimensional periodic lattice (mass-spring system) containing a disorder that is itself a one-dimensional periodic lattice. The concepts of the propagation constant and wave-receptance function are used to determine the receptances of the component systems, and these are used to set up a simple frequency equation. An accurate root-searching computer program has been used to find the natural frequencies and corresponding modes of particle displacement. Some computed results are shown to demonstrate the capability of the method and program. Special attention is given to modes that occur in the frequency "forbidden" zone, and receptance methods are used to derive formulas for the frequencies of systems with a single-mass disorder. The wider usefulness of the method is briefly discussed.

Mead, D. J., and Yaman, Y., 1990. "The Harmonic Response of Uniform Beams on Multiple Linear Supports: A Flexural Wave Analysis,' Journal of Sound and Vibration, Vol. 141, pp. 465-484.

A wave approach is developed for the exact analysis of the harmonic response of uniform finite beams on multiple supports. The beam may be excited by single- or multiple-point harmonic forces or moments; its supports may have general linear characteristics that may include displacement-rotation coupling. Use is made of the harmonic response function for an infinite beam subjected to a single-point harmonic force or moment. The unknowns of the finite beam problem are the support reaction forces/mo- 
ments and the magnitudes of four waves reflected from the ends of the beam. Equations are presented for the response of a single-bay beam with various support conditions and subjected to single-point harmonic excitation. The same equations, but with the simple addition of further straightforward terms on the right-hand side, are used for multipoint excitation. The effects of damping are easily incorporated. Equations for multisupported beams are also presented together with illustrative computed frequency-response curves. Natural frequencies have been calculated by finding resonance frequencies of lightly damped beams. These compare impeccably with the results of other investigators.

Mead, D. J., and Yaman, Y., 1991, "The Response of Infinite Periodic Beams of Point Harmonic Forces: A Flexural Wave Analysis," Journal of Sound and Vibration, Vol. 144, pp. 507-30.

An exact analysis is presented of the vibration response of an infinite beam on periodic supports, subjected to a transverse harmonic point force. The supports must all be the same and can be simply supported or be generally linear with elastic, inertial, and dissipative properties. The total response is found as the sum of the flexural wave fields generated by the applied force and the infinite number of support reaction forces and moments. The concept of phased arrays of forces and moments is used to sum the support-generated wave fields. This utilizes the propagation constants of free-wave motion in the periodic beam. Equations for either four, six, or eight of the unknown complex reaction (depending on the nature of the supports) are set up and solved numerically. This finite number is sufficient to permit the calculation of the beam displacement at any point and of all the other reactions. Some computed values of the beam direct receptance are presented to demonstrate its variation with forcing frequency, the effect of the location of the excitation force, and the effect of changing the elastic properties of the supports.

Mead, D. J., Zhu, D. C., and Bardell, N. S., 1988, "Free Vibration of an Orthogonally Stiffened Flat Plate," Journal of Sound and Vibration. Vol. 27, pp. 19-48.

A flat plate, reinforced by a regular orthogonal array of uniform beams, is analyzed by using techniques developed for studying wave propagation in twodimensional periodic structures. A "plane-wave" type of motion is considered that may be characterized by different propagation phase constants in the $x$ - and $y$-directions. The hierarchical finite element method is used to set up the governing equations of free wave motion, and these are then solved as an eigenvalue problem for the frequencies at which particular waves will propagate. Plots of phase constant surfaces vs. frequency are presented for a number of different plate-beam configurations. Excellent agreement is found between some of these and the results of earlier investigators. When the plate is supported by flexible beams in both directions, wave propagation is found to commence at zero frequency. At higher frequencies alternating (and overlapping) attenuation and propagation bands occur. The nature and explanations of these are discussed. Wave speed surfaces vs. frequency are also presented and these give insight into the critical coincidence frequencies of the plate under acoustic excitation.

Meirovitch, L., and Engels, R. C., 1977, "Response of Periodic Structures by the Z-Transform Method," AIAA Journal, Vol. 15, pp. 167-174.

Periodic structures are defined as structures consisting of identical substructures connected to each other in an identical manner. The response of periodic structures to harmonic excitation can be described by a matrix difference equation. The solution of the matrix difference equation can be obtained by the $Z$-transform method and it yields the response to both end conditions and external excitations. The method developed necessitates the eigenvalues of the transfer matrix for a typical substructure, so that the procedure is capable of analyzing a periodic structure with the same computational effort necessary to analyze a single substructure. An added advantage is derived from the fact that the method does not require the eigenvectors of the transfer matrix.

Meirovitch, L., and Engels, R. C., 1978, "Response of Almost Periodic Structures," Journal de Mecanique Appliquee, Vol. 2, pp. 197-210.

Periodic structures are defined as structures consisting of identical substructures connected to each other in an identical manner. If the system parameters differ slightly from one substructure to another, then the structure becomes almost periodic. An efficient method using a perturbation technique to derive the response of an almost-periodic structure is presented. The procedure reduces the solution to a sequential application of the basic algorithm for periodic structures developed by these authors. The method retains all the advantages of the method for periodic structures and reduces to it when the system parameters become identical for each substructure.

Mester, S. S., 1992, "Wave Propagation in Periodic Structures with Imperfections," Master's Thesis, 
Graduate School-New Brunswick Rutgers, The State University of New Jersey.

Extensive work has been done on the vibration characteristics of perfectly periodic structures. The purpose of this investigation is to begin to determine the effects of disorder in one-dimensional and twodimensional periodic structures. From the state of the art review given, disorder has been found to lead to localization on one-dimensional periodic structures in previous analyses. It is important to understand localization because it causes energy to be concentrated near the disorder. Various biperiodic structures are first analyzed in this work. The dispersion relations are found to have two branches, one for each variation in the biperiodic element. Random variable dispersion relations along with the probability distribution function of the dispersion relations have been found. Applications of interest include structural dynamics and control of structures. These applications are discussed here.

Miller, D. W., and von Flotow, A., 1989, "A Travelling Wave Approach to Power Flow in Structural Networks," Journal of Sound and Vibration, Vol. 128, pp. 145-162.

A structural network is an assemblage of slender one-dimensional members. Each member is demarcated, by definition, by two junctions. A junction may involve only one member (a termination) or maybe an interconnection of many members. In this article the dynamics of such networks are investigated computationally and theoretically. The focus of the analysis is on elastic disturbance propagation and power flow in the network. Spatially local models are assembled into a global, frequency domain description. This global description of the response is then investigated for local and global power flow. The procedure serves to identify disturbance transmission paths and to choose and evaluate control procedures, both active and passive. The techniques are demonstrated by application to several examples.

Miller, D. W., Hall, S. R., and von Flotow, A., 1989, "Optimal Control of Power Flow at Structural Junctions," Conference Proceedings of the 1989 American Control Conference, Vol. 1, pp. 212-220.

Several techniques are described for deriving optimal feedback compensators for structural waveguides at junctions. A frequency-dependent cost functional, composed of power flow and control effort, is minimized. Control of power flow, by modifying junction reflection and transmission properties, enables selective absorption of incoming vibrational power. Noncausal, causal fixed form, and Weiner-Hopf feedback solutions are derived.
These solutions, including a positive real approximation to the Weiner-Hopf solution, are illustrated through an extensive example for the free end of a dispersive Bernoulli-Euler beam.

Nagem, R., and Williams, J., 1989, "Dynamic Analysis of Large Space Structures Using Transfer Matrices and Joint Coupling Matrices,"' Mechanics of Structures and Machines, Vol. 17, pp. 349-371.

Linear dynamic analysis of lattice structures using transfer matrices and joint coupling matrices is presented. A lattice structure is defined as a network of one-dimensional members that are connected by joints. Two examples are considered to illustrate how transfer matrices and joint coupling matrices may be used to compute natural frequencies of vibration. These two examples indicate that the transfer matrix and joint coupling matrix analysis is numerically accurate over a wide range of frequencies and becomes increasingly efficient, compared to the finite element method, as the frequency increases. Some suggestions for further improvements in computational efficiency and some comments about applicability to numerical analysis of wave propagation problems are given.

Noor, A. K., Anderson, M. S., and Greene, W. H., 1978, "Continuum Models for Beam-Like and PlateLike Lattice Structures,' AIAA Journal, Vol. 16, pp. 1219-1228.

A simple, rational approach is presented for developing continuum models for large repetitive beamlike and plate-like lattices with arbitrary configurations subjected to static, thermal, and dynamic loadings. The continuum models for these structures are shear flexible beams and plates. They account for local effects in the repeating element of the actual structure and are characterized by their thermoelastic strain and kinetic energies from which the equations of motion and constitutive relations can be derived. The procedure for developing the expressions for thermoelastic strain and kinetic energies of the continuum involves introducing basic assumptions regarding the variation of the temperature, displacement, and strain components in one or two directions (for plate-like and beam-like lattices) and obtaining effective thermoelastic and dynamic coefficients of the continuum in terms of material properties and geometry of the original lattice structure. The high accuracy of the continuum models developed is demonstrated by means of numerical examples.

Noor, A. K., and Mikulas, M. M., 1988, "Continuum Modeling of Large Lattice Structures: Status and Projections," NASA Technical Report 2767. 
The status and some recent developments of continuum modeling for large repetitive lattice structures are summarized. Discussion focuses on a number of aspects including definition of an effective substitute continuum; characterization of the continuum model; and the different approaches for generating the properties of the continuum, namely, the constitutive matrix, and matrix of mass densities, and the matrix of thermal coefficients. Also a simple approach is presented for generating the continuum properties. The approach can be used to generate analytic and/or numerical values of the continuum properties.

Pierre, C., 1988, "Mode Localization and Eigenvalue Loci Veering Phenomena in Disordered Structures," Journal of Sound and Vibration, Vol. 126, pp. 485502.

An investigation of the effects of disorder on the modes of vibration of nearly periodic structures is presented. It is shown that, in structures with close eigenvalues, small structural irregularities result in both strong localization of the mode shapes and abrupt veering away, or mutual repulsion, of the loci of the eigenvalues when these are plotted against a parameter representing the disorder in the system. Perturbation methods for the eigenvalue problem are applied to predict the occurrence of strong localization and eigenvalue loci veering, which are shown to be two manifestations of the same phenomenon. Also, perturbation methods that handle the dramatic effects of small disorder are developed to analyze eigenvalue loci veering and strong localization. Two representative disordered nearly periodic structures are studied: a mistuned assembly of coupled oscillators and a multispan beam with irregular spacing of the supports.

Pierre, C., 1990, "Weak and Strong Vibration Localization in Disordered Structures: A Statistical Investigation," Journal of Sound and Vibration, Vol. 139, pp. 111-132.

A statistical investigation of the effects of disorder on the dynamics of one-dimensional nearly periodic structures is presented. The problem of vibration propagation from a local source of excitation is considered. Although for the ordered infinite system there exists a frequency passband for which the vibration propagates without attenuation, the introduction of disorder results in an exponential decay of the amplitude for all excitation frequencies. Analytical expressions for the localization factors (the exponential decay constants) are obtained in the two limiting cases of weak and strong internal coupling, and the degree of localization is shown to depend upon the disorder to coupling ratio and the excitation frequency.
Pierre, C., Tang, D. M., and Dowell, E. H., 1987, "Localized Vibrations of Disordered Multispan Beams-Theory and Experiment," AIAA Journal, Vol. 25, pp. 1249-1257.

The localization of the free modes of vibration of disordered multispan beams is investigated, both theoretically and experimentally. It is shown that small deviations of the span lengths from an ideal value may have drastic effects on the dynamics of the system, by inhibiting the propagation of vibrations in the structure. Emphasis is placed on the development of a perturbation method that allows one to obtain the localized modes of vibration of the disordered system without a global eigenvalue analysis of the entire system. Such a perturbation analysis is cost effective and accurate. More importantly, it provides physical insight into the localization phenomenon, and allows one to formulate a criterion that predicts the occurrence of localized modes. Also, an experiment is described that has been carried out to verify the existence of localized modes for disordered two-span beams. Theoretical and experimental results are compared in detail and excellent agreement is found, thus confirming the existence of localized modes.

Pines, D. J., and von Flotow, A., 1990, "Active Control of Bending Wave Propagation at Acoustic Frequencies," Journal of Sound and Vibration, Vol. 142, pp. 391-412.

An analytical and experimental investigation of the possibility of actively blocking the propagation of bending waves along a uniform beam is described. The investigation has the form of a case study: a thin brass plate-beam is used, it is excited with a short duration impulse, and the resulting disturbance spreads dispersively as it travels along the beam. A short portion of the beam is used as an active block. Strain gauge sensors are used to drive thin piezoceramic bending moment actuators through a dynamic compensator. The compensation is designed in the frequency domain with reference to the beam equation, but independent of boundary conditions. The analytical work described includes the nominal design and its performance, the performance degradation due to modeling errors, and the performance degradation due to approximate implementation of the dynamic compensator. The laboratory implementation, in which analog electronics were used for the compensator, essentially verifies some of the sensitivity predictions.

Soong, T. T., and Bogdanoff, J. L., 1963, "On the Natural Frequencies of a Disordered Linear Chain of 
$N$ Degrees of Freedom," International Journal of Mech Science, Vol. 5, pp. 237-265.

Statistical properties of the natural frequencies of a linear chain having $N$ degrees of freedom are derived assuming the physical properties of its elements or parameters are known only in a stochastic sense. The method of derivation is based upon a transfer matrix scheme together with a perturbationtype expansion. It is found that the top few natural frequencies have values that are very sensitive to the parameter variations whereas the lowest few are insensitive to these variations.

Soong, T. T., and Bogdanoff, J. L., 1964, "On the Impulsive Admittance and Frequency Response of a Disordered Linear Chain of $N$ Degrees of Freedom,'” International Journal of Mech Science, Vol. 6, pp. 225-237.

Analytical and numerical results are presented on the statistics of the impulsive admittance and frequency response of a linear chain of $N$ degrees of freedom when the physical properties of its elements are only known in a stochastic sense. These results illustrate, on a quantitative basis, the significance of stochastic parameters on the dynamical behavior of engineering systems, and thereby enable us to rationally establish acceptable tolerance on system components to achieve certain accuracy in predicted system performances.

von Flotow, A., 1986, "Disturbance Propagation in Structural Networks," Journal of Sound and Vibration, Vol. 106, pp. 433-450.

A structural network is taken to be an assemblage of slender structural members (beams, cables, and rods) connected to each other at structural junctions. The junctions may include flexible bodies that in this work are restricted to those whose dynamics are described by a finite set of ordinary differential equations. Elastic disturbances in such a network are calculated in terms of propagation concepts. Members are described in the frequency domain by the propagation coefficients of their intrinsic wavemodes, junctions by frequency-dependent wavemode reflection and transmission coefficients, grouped in the junction scattering matrix. Component impulse responses are calculated by a combination of analysis and application of the fast Fourier transform algorithm. Network time responses are synthesized by convolution of component impulse responses. A consistent analytical framework is constructed within which descriptions of various member types and junctions can be accommodated. von Flotow, A., 1986, "Traveling Wave Control for Large Spacecraft Structures," Journal of Guidance, Control, and Dynamics, Vol. 9, pp. 461-468.

This article introduces the point of view that elastic deformations in large spacecraft structures may be aptly viewed in terms of propagating disturbances. Because the main topic of this article is the control concepts, which result from such a view point, the required structural dynamic description in terms of traveling disturbances is described only briefly, with reference to previously published works. The active control of these structures is approached from the point of view of actively modifying the natural disturbance propagation paths. Elastic energy is shunted into unimportant portions of the structure or is absorbed by an active "wave absorber." Several computational examples demonstrate the remarkable theoretical performance achievable by propagation-based controllers.

von Flotow, A., and Schaefer, B., 1986, "Wave-Absorbing Controllers for a Flexible Beam," Journal of Guidance, Control, and Dynamics, Vol. 9, pp. 673680.

This article describes theoretical and experimental work performed on the modeling and vibration control of a hanging flexible beam. The synthesis and laboratory implementation of low authority controllers based upon feed back of local velocity to actuator force has been the subject of previous studies. The study extends this work with the design and laboratory implementation of low authority controllers based upon concepts of disturbance propagation and reflection. Control forces are applied to the lower end of the hanging beam. Compensators are derived that feed back local deflection and slope to control force and moment with the goal of minimizing the reflection of energy at the lower end. Several of these compensators are approximated by analog electronic filters for laboratory implementation. The performance of these wave-absorbing compensators is compared with that of velocity feedback.

Wittrick, W. H., and Williams, F. W., 1971, "A General Algorithm for Computing Natural Frequencies of Elastic Structures," Quarterly Journal of Mechanics and Applied Mathematics, Vol. 24, pp. 263-284.

The authors present and prove an algorithm for determining the natural undamped frequencies of vibration of any linearly elastic structure if its dynamic stiffness matrix $K$ (omega) corresponding to any finite set of displacements $D$ is known. In general $K$ (omega) is not a linear function of omega ${ }^{2}$, and methods that are available for solving linear eigenvalue problems are inapplicable. The algorithm is valid for systems with either a finite or infinite 
number of degrees of freedom. It enables one to calculate how many natural frequencies lie below any chosen frequency, without determining them, and hence to converge on any required natural frequency to any specified accuracy.

Zhang, X. M., and Zhang, W. H., 1991, "The Reduction of Vibrational Energy Flow in a Periodically Supported Beam,' Journal of Sound and Vibration, Vol. 151, pp. 1-7.

The reduction of vibrational energy flow in a spatially, periodically supported beam is studied. The influence of the excitation location on the transmitted and travelling energy flows is investigated. For different propagation bands, the vibrational energy transmitted into the beam can be reduced to a minimum by suitably adjusting the excitation location. No matter where the beam is excited, the energy flows along the beam in the positive and negative directions are the same in magnitude and equal to one-half of the transmitted power. The effect of structural damping on the energy flow is also studied. In this case the periodic beam is excited at the midpoint of one bay. The transmitted power is effectively controlled by damping near the bounding frequencies of the odd propagation bands. The energy flow is effectively decreased by damping in the propagation bands.

Zhong, W. X., and Williams, F. W., 1991, "On the Localization of the Vibration Mode of a Substructural Chain-Type Structure," Proceedings of the Institution of Mechanical Engineers, Part C: Mechanical Engineering Science, Vol. 205, pp. 281-288.

The localization phenomenon for high-frequency vibration modes of imperfectly repetitive structures is considered by using an analogy between computational structural mechanics theory and optimal control theory. In particular, the simplectic matrix method of control theory is applied to the dynamic external stiffness matrix of a typical repeating substructure. The eigensolution of the simplectic matrix for the case where the substructures are connected by a single line of springs is solved analytically. This gives the passband for the frequency of the travelling waves along the substructural chain and the corresponding eigenmode. Hence it is shown how the influence of local disturbances of the substructural chain on the behavior of the whole chain can be considered analytically, thus explaining the mechanism of the localization of high-frequency vibration modes. Numerical examples are therefore not needed, although a few are introduced solely to confirm the correctness of conclusions already drawn from the analytical method.
Ziegler, F., 1977, "Wave Propagation in Periodic and Distorted Layered Composite Elastic Materials,"' International Journal of Solids and Structures, Vol. 13, pp. 293-305.

A powerful complex transfer matrix approach to wave propagation perpendicular to the layering of a composite of periodic and disordered structure is worked out showing propagating and stopping bands of time-harmonic waves and the singular cases of standing waves. A state ratio of left and right going plane waves is defined and interpreted geometrically in the complex plane in terms of fixed points and flow lines. For numerical considerations and extension of the approach to higher dimensional problems, a continued fraction expansion of the state ratio mapping is presented. Impurity modes of wave propagation in composites with widely spaced impurity cells of different elastic materials are discussed. Stopping bands in the frequency spectrum of global waves in fully disordered composites are found to exist in the range of frequencies corresponding to common gaps in the spectrum of constituent regular periodic composites that are constructed from the cells of the disordered system. For those frequencies, waves propagate only a (short) finite distance and are therefore strongly localized modes in a composite of fairly large extent.

\section{REFERENCES}

Anderson, M. S., 1981, "Buckling of Periodic Lattice Structures," AIAA Journal, Vol. 19, pp. 782-788.

Anderson, M. S., 1982, "Vibration of Prestressed Periodic Lattice Structures,"' AIAA Journal, Vol. 20, pp. 551-555.

Bardell, N., and Mead, D., 1989a, "Free Vibration of an Orthogonally Stiffened Cylindrical Shell, Part I: Discrete Line Simple Supports," Journal of Sound and Vibration, Vol. 134, pp. 29-54.

Bardell, N., and Mead, D., 1989b, "Free Vibration of an Orthogonally Stiffened Cylindrical Shell, part II: Discrete General Stiffeners,' Journal of Sound and Vibration, Vol. 134, pp. 55-72.

Bendiksen, O. O., 1987, Mode Localization Phenomena in Large Space Structures AIAA Journal, Vol. 25, pp. 1241-1248.

Bennighof, J. K., and Meirovitch, L., 1989, “Active Suppression of Traveling Waves in Structures," Journal Guidance, Vol. 12, pp. 555-567.

Bouzit, D., and Pierre, C., 1993, "An Experimental Investigation of Vibration Localization in Disordered Multi-Span Beams," Technical Report AIAA-93-1487-CP, AIAA.

Cai, G., and Lin, Y., 1990, "Localization of Wave Propagation in Disordered Periodic Structures," AIAA Journal, Vol. 29, pp. 1555-1574. 
Cai, G., and Lin, Y., 1991, "Wave Propagation and Scattering in Structural Networks," Journal of Engineering Mechanics, Vol. 117, pp. 1555-1574.

Cha, P. D., and Pierre, C., 1991, "Vibration Localization by Disorder in Assemblies of Monocoupled, Multimode Component Systems," Journal of Applied Mechanics, Vol. 58 (91-APM-27).

Denke, P. H., Eide, G. R., and Pickard, J., 1975, "Matrix Difference Equation Analysis of Vibrating Periodic Structures," AIAA Journal, Vol. 13, pp. 160-166.

Engels, R., 1980, "Response of Infinite Periodic Structures," Journal of Sound and Vibration, Vol. 69, pp. 181-197.

Engels, R., and Meirovitch, L., 1978a, "Response of Periodic Structures by Modal Analysis," Journal of Sound and Vibration, Vol. 56, pp. 481-493.

Engels, R., and Meirovitch, L., 1978b, "Simulation of Continuous Systems by Periodic Structures," Journal of Applied Mechanics, Vol. 45, pp. 385-392.

Feather, N., 1961, An Introduction to the Physics of Vibrations and Waves, Edinburgh University Press.

Ghatak, A. K., and Kothari, L. S., 1972, An Introduction to Lattice Dynamics, Addison-Wesley Publishing Company, Boston.

Hagwood, N. W., Chung, W. H., and von Flotow, A., 1990, "Modeling of Piezoelectric Actuator Dynamics for Active Structural Control," Technical Report, MIT.

Hodges, C., 1982, "Confinement of Vibration by Structural Irregularity,' Journal of Sound and Vibration, Vol. 82, pp. 411-424.

Huang, W. hu, 1982, "Vibration of Some Structures with Periodic Random Parameters," AIAA Journal, Vol. 20, pp. 1001-1008.

Keane, A. J., and Price, W. G., 1989, "On the Vibrations of Mono-Coupled Periodic and Near-Periodic Structures," Journal of Sound and Vibration, Vol. 128, pp. 423-450.

Levine-West, M. B., and Salama, M. A., 1992, "Mode Localization Experiments on Ribbed Antenna," in The 33rd AIAA/ASME/ASCE/AHS/ASC Structures, Structural Dynamics and Materials Conference, Dallas, TX, April 13-15.

Lin, Y. K., and McDaniel, T. J., 1969, "Dynamics of Beam-Type Periodic Structures," Journal of Engineering for Industry, Vol. 91, pp. 1133-1141, Ser. B.

McDaniel, T., and Carroll, M., 1982, "Dynamics of Bi-Periodic Structures," Journal of Sound and Vibration, Vol. 81, pp 311-335.

Mead, D., 1973, "A General theory of Harmonic Wave Propagation in Linear Periodic Systems with Multiple Coupling," Journal of Sound and Vibration, Vol. 27, pp. 235-260.

Mead, D., 1975a, "Wave Propagation and Natural Modes in Periodic Systems: I. Mono-coupled Systems,' Journal of Sound and Vibration, Vol. 40, pp. $1-18$.
Mead, D., 1975b, "Wave Propagation and Natural Modes in Periodic Systems, with and without Damping," Journal of Sound and Vibration, Vol. 40, pp. 19-39.

Mead, D., 1985, "Wave Propagation in Timoshenko Beams," Strojnicky Casopis, Vol. 36, pp. 556-585.

Mead, D., 1986, “A New Method of Analyzing Wave Propagation in Periodic Structures; Applications to Periodic Timoshenko Beams and Stiffened Plates,' Journal of Sound and Vibration, Vol. 104, pp. 9-27.

Mead, D., and Bansal, A., 1978, "Mono-Coupled Periodic Systems with a Single Disorder: Free Wave Propagation," Journal of Sound and Vibration, Vol. 61, pp. 481-496.

Mead, D., and Bardell, N., 1986, "Free Vibration of a Thin Cylindrical Shell with Discrete Axial Stiffeners," Journal of Sound and Vibration, Vol. 111, pp. 229-250.

Mead, D., and Bardell, N., 1987, "Free Vibration of a Thin Cylindrical Shell with Periodic Circumferential Stiffeners," Journal of Sound and Vibration, Vol. 115, pp. 499-520.

Mead, D., and Lee, S., 1984, "Receptance Methods and the Dynamics of Disordered One-Dimensional Lattices," Journal of Sound and Vibration, Vol. 92 , pp. 427-445.

Mead, D., and Yaman, Y., 1990, "The Harmonic Response of Uniform Beams on Multiple Linear Supports: A Flexural Wave Analysis," Journal of Sound and Vibration, Vol. 141, pp. 465-484.

Mead, D., and Yaman, Y., 1991, "The Response of Infinite Periodic Beams to Point Harmonic Forces: A Flexural Wave Analysis," Journal of Sound and Vibration, Vol. 144, pp. 507-530.

Mead, D., Zhu, D., and Bardell, N., 1988, "Free Vibration of an Orthogonally Stiffened Flat Plate," Journal of Sound and Vibration, Vol. 127, pp. 1948.

Meirovitch, L., and Engels, R. C., 1977, "Response of Periodic Structures by the Z-Transform Method," AIAA Journal, Vol. 15, pp. 167-174.

Meirovitch, L., and Engels, R. C., 1978, "Response of Almost Periodic Structures," Journal de Mecanique Appliquee, Vol. 2, pp. 199-210.

Mester, S. S., 1992, "Wave Propagation in Periodic Structures with Imperfections," Master's thesis, New Brunswick Rutgers, The State University of New Jersey.

Miller, D., and von Flotow, A., 1989, "A Traveling Wave Approach to Power Flow in Structural Networks,' Journal of Sound and Vibration, Vol. 128, pp. 145-162.

Miller, D. W., Hall, S. R., and von Flotow, A. H., 1989, Optimal Control of Power Flow At Structural Junctions, American Control Conference, Pittsburgh.

Muller, D., 1956, "A Method for Solving Algebraic Equations Using an Automatic Computer," Mathematical Tables Aids Computers, Vol. 10. 
Nagem, R. J., and Williams, J. H., Jr., 1989, “Dynamic Analysis of Large Space Structures Using Transfer Matrices and Joint Coupling Matrices,' Mechanical Structures and Machines, Vol. 17, pp. 349-371.

Noor, A. K., Anderson, M. S., and Greene, W. H., 1978, "Continuum Models for Beam- and Platelike Lattice Structures," AIAA Journal, Vol. 16, pp. 1219-1228.

Noor, A. K., and Mikulas, M. M., Jr., 1988, "Continuum Modeling of Large Lattice Structures, Status and Projections," Technical Report 2767, NASA.

Pestel, E. C., and Leckie, F. A., 1963, Matrix Methods in Elasto-mechanics, McGraw-Hill, New York.

Pierre, C., 1988, “Mode Localization and Eigenvalue Loci Veering Phenomena in Disordered Structures," Journal of Sound and Vibration, Vol. 126, pp. 485-502.

Pierre, C., 1990, “Weak and Strong Vibration Localization in Disordered Structures: A Statistical Investigation," Journal of Sound and Vibration, Vol. 139, pp. 111-132.

Pierre, C., Tang, D. M., and Dowell, E. H., 1987, "Localized Vibrations of Disordered Multispan Beams: Theory and Experiment," AIAA Journal, Vol. 25, pp. 1249-1257.

Pines, D., and von Flotow, A., 1988, “Active Control of Bending Wave Propagation at Acoustic Frequencies," Technical Report, MIT.

Soong, T. T., and Bogdanoff, J. L., 1963, "On the Natural Frequencies of a Disordered Linear Chain of $n$ Degrees of Freedom, International Journal of Mechanical Science, Vol. 5, pp. 237-265.

Soong, T. T., and Bogdanoff, J. L., 1964, "On the Impulsive Admittance and Frequency Response of a Disordered Linear Chain of $n$ Degrees of Freedom," International Journal of Mechanical Science, Vol. 6, pp. 225-237.

von Flotow, A., 1986a, "Disturbance Propagation in Structural Networks," Journal of Sound and Vibration, Vol. 106, pp. 433-450.

von Flotow, A., 1986b, "'Traveling Wave Control for Large Spacecraft Structures,' Journal of Guidance, Control, and Dynamics, Vol. 9, pp. 462-468.

von Flotow, A., and Schafer, B., 1986, "Wave-Absorbing Controllers for a Flexible Beam," Journal of Guidance, Vol. 9, pp. 673-680.

Wittrick, W. H., and Williams, F. W., 1971, "A General Algorithm for Computing Natural Frequencies of Elastic Structures," Quarterly Journal of Mechanics and Applied Mathematics, Vol. 14.

Zhang, X., and Zhang, W., 1991, "The Reduction of Vibrational Energy Flow in a Periodically Supported Beam," Journal of Sound and Vibration, Vol. 151, pp. 1-7.

Zhong, W. X., and Williams, F. W., 1991, "On the Localization of the Vibration Mode of a Sub-Structural Chain-Type Structure," Proceedings of the Institution of Mechanical Engineering, Vol. 205, pp. 289-288.

Ziegler, F., 1977, "Wave Propagation in Periodic and Disordered Layered Composite Elastic Materials," International Journal of Solids and Structures, Vol. 13, pp. 293-305. 

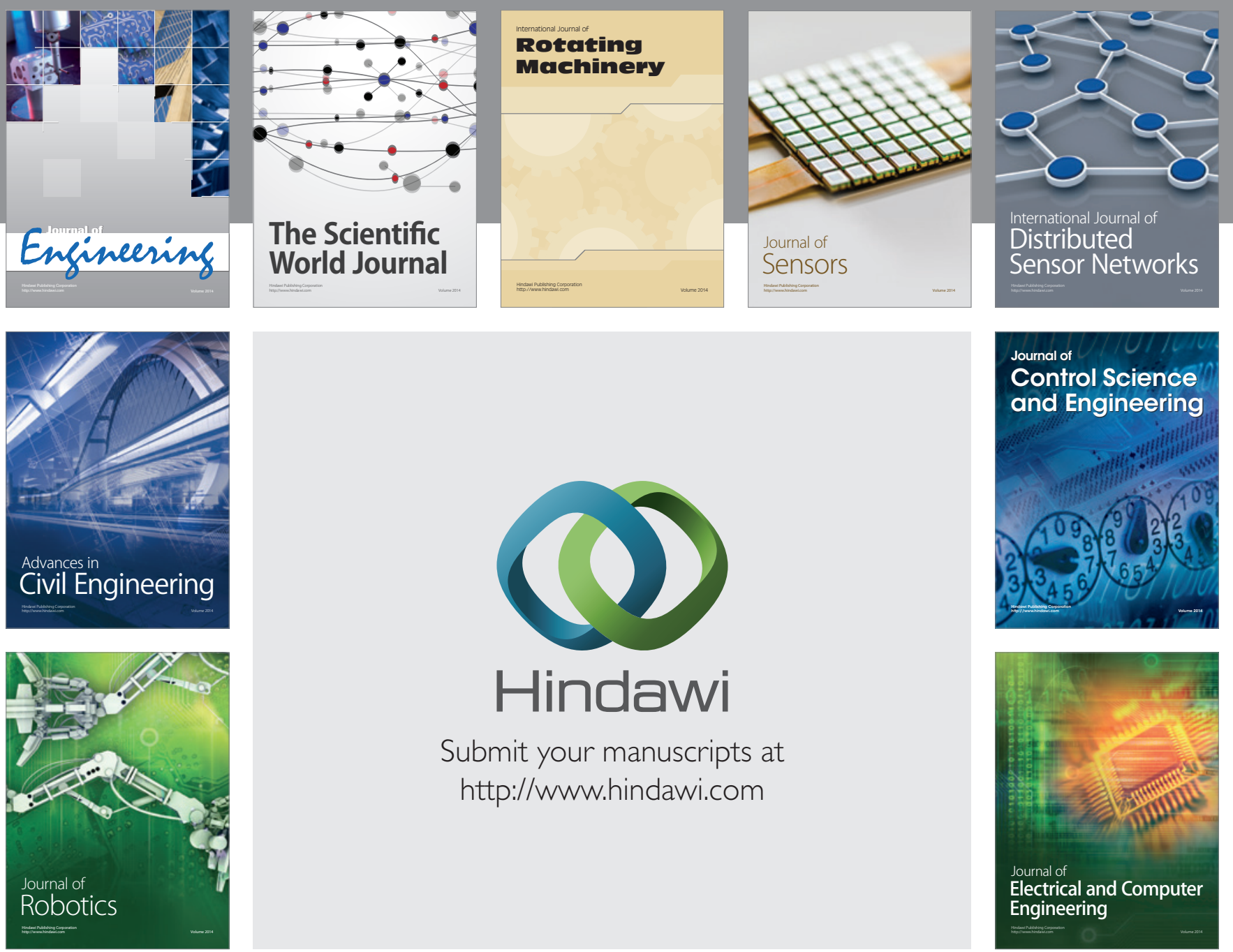

Submit your manuscripts at

http://www.hindawi.com
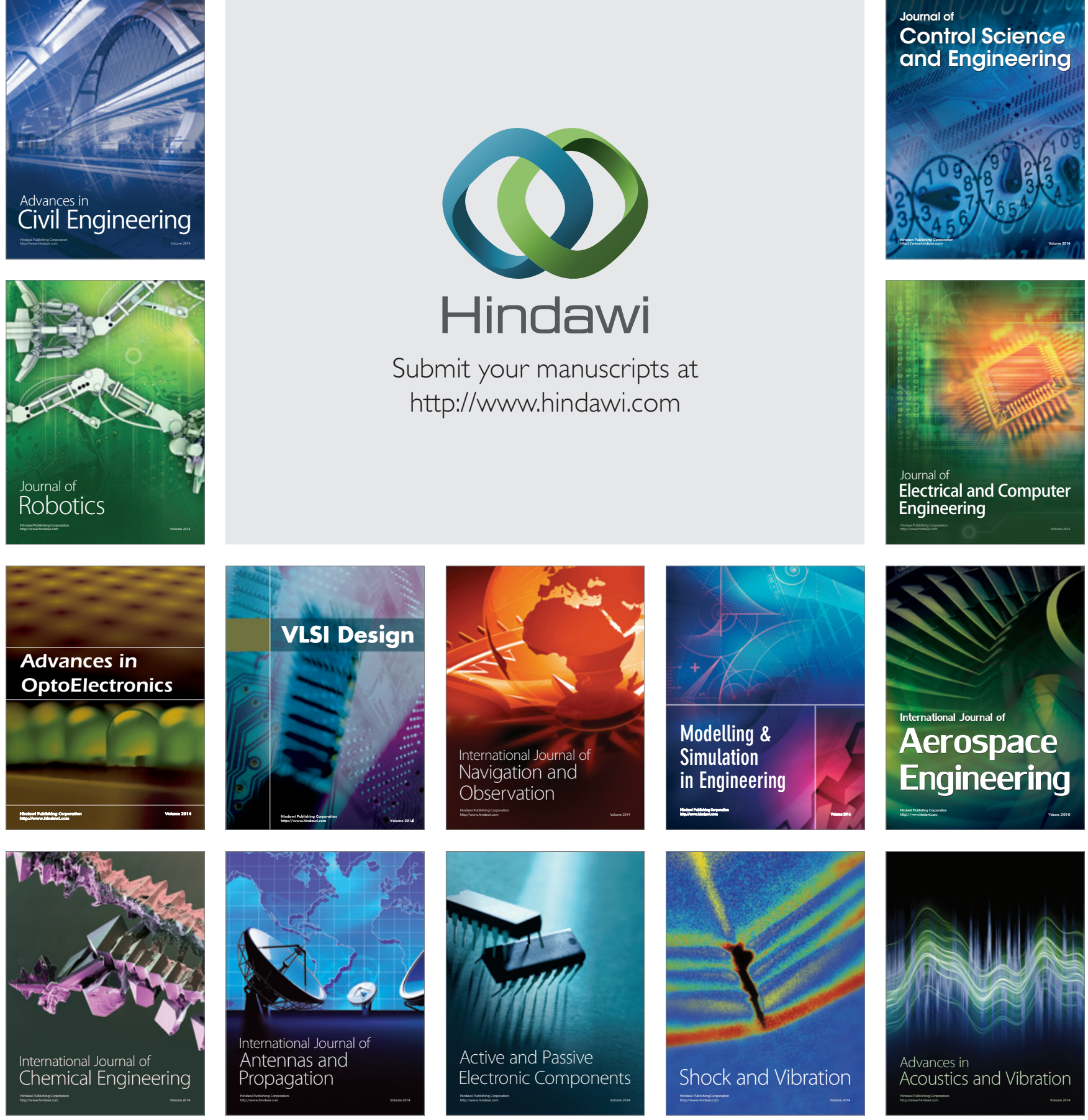